\title{
biomolecules
}

ISSN 2218-273X

www.mdpi.com/journal/biomolecules/

Article

\section{HIV-1 Recruits UPF1 but Excludes UPF2 to Promote Nucleocytoplasmic Export of the Genomic RNA}

\author{
Lara Ajamian 1,2, Karen Abel 1,3, Shringar Rao 1,3, Kishanda Vyboh 1,3, \\ Francisco García-de-Gracia ${ }^{4}$, Ricardo Soto-Rifo ${ }^{4}$, Andreas E. Kulozik ${ }^{5,6}$, \\ Niels H. Gehring ${ }^{7}$ and Andrew J. Mouland 1,2,3,*
}

1 HIV-1 RNA Trafficking Laboratory, Lady Davis Institute for Medical Research-Sir Mortimer B. Davis Jewish General Hospital, Montréal QC H3T 1E2, Canada;

E-Mails: lara.ajamian@mail.mcgill.ca (L.A.); karen_abel@gmx.de (K.A.); shringar.rao@mail.mcgill.ca (S.R.); kishanda.vyboh@mail.mcgill.ca (K.V.)

2 Department of Medicine, Division of Experimental Medicine, McGill University, Montréal QC H3A 2B4, Canada

3 Department of Microbiology and Immunology, McGill University, Montréal QC H3T 1E2, Canada

4 Laboratory of Molecular and Cellular Virology, Virology Program, Biomedical Sciences Institute, Faculty of Medicine, Universidad de Chile, Independencia 8389100, Santiago, Chile;

E-Mails: franciscogarcia96@gmail.com (F.G.-G.); rsotorifo@med.uchile.cl (R.S.-R.)

5 Department of Pediatric Oncology, Hematology and Immunology, Heidelberg 69120, Germany; E-Mail: andreas.kulozik@med.uni-heidelberg.de

6 European Molecular Biology Laboratory, Partnership Unit, University of Heidelberg Molecular Medicine, Heidelberg 69117, Germany

7 Institute for Genetics, University of Cologne, Cologne 50674, Germany;

E-Mail: ngehring@uni-koeln.de

* To whom correspondence should be addressed; E-Mail: andrew.mouland@mcgill.ca; Tel.: +1-514-340-8260; Fax: +1-514-340-7502.

Academic Editor: André Gerber

Received: 7 July 2015 / Accepted: 16 September 2015 / Published: 20 October 2015

Abstract: Unspliced, genomic HIV-1 RNA (vRNA) is a component of several ribonucleoprotein complexes (RNP) during the viral replication cycle. In earlier work, we demonstrated that the host upframeshift protein 1 (UPF1), a key factor in nonsense-mediated mRNA decay (NMD), colocalized and associated to the viral structural protein Gag during viral egress. In this work, we demonstrate a new function for UPF1 in the regulation of vRNA nuclear export. 
We establish that the nucleocytoplasmic shuttling of UPF1 is required for this function and demonstrate that UPF1 exists in two essential viral RNPs during the late phase of HIV-1 replication: the first, in a nuclear export RNP that contains Rev, CRM1, DDX3 and the nucleoporin p62, and the second, which excludes these nuclear export markers but contains Gag in the cytoplasm. Interestingly, we observed that both UPF2 and the long isoform of UPF3a, UPF3aL, but not the shorter isoforms UPF3aS and UPF3b, are excluded from the UPF1-Rev-CRM1-DDX3 complex as they are negative regulators of vRNA nuclear export. In silico protein-protein docking analyses suggest that Rev binds UPF1 in a region that overlaps the UPF2 binding site, thus explaining the exclusion of this negative regulatory factor by HIV-1 that is necessary for vRNA trafficking. This work uncovers a novel and unique regulatory circuit involving several UPF proteins that ultimately regulate vRNA nuclear export and trafficking.

Keywords: HIV-1; UPF1; ribonucleoprotein; nuclear RNA export; viral evasion; nonsense mediated decay

\section{Introduction}

The nucleocytoplasmic export of macromolecules (RNA and protein) and RNA-protein (RNP) complexes is one of the critical steps necessary to ensure normal cellular function. The study of retroviral RNA export has derived a great deal of information on this highly regulated process that represents a key step in the replication cycle that could serve as a viable therapeutic target [1,2]. For instance, the recruitment of viral and host factors to the unspliced viral genomic RNAs (vRNA is used herein) helps this molecule to overcome nuclear retention and promote nucleocytoplasmic export $[3,4]$. Bypassing host RNA surveillance machineries that target and clear unspliced and intron-containing, aberrantly spliced RNA substrates leads to the preservation of viral genomic RNAs and guarantees structural viral protein synthesis once they arrive in the cytoplasm [5-7].

While the nuclear RNA export factor 1 (NXF1) is required for the constitutive export of the 2-kb, completely-spliced viral RNA species [8], the 9-kb, unspliced, vRNA and the 4-kb, singly-spliced RNAs harbor the cis-acting Rev-responsive element (RRE) that are bound by the viral protein Rev to program nucleocytoplasmic RNA trafficking [9]. Many studies have highlighted the requirement for various host proteins in these transport processes. For example, eukaryotic translation initiation factor 5A (eIF5A) acts as an adaptor for Rev and the mammalian nuclear export factor Chromosomal Maintenance 1 (CRM1) [10,11]. RNA helicases such as DEAD box protein 1 and 3 (DDX1, 3) promote Rev-mediated export via interaction with CRM1 and Rev [12]. The Src-associated protein in mitosis, $68 \mathrm{kDa}$ (Sam68), is not only required for Rev-dependent export, but is also involved in exporting RRE-containing RNAs in a Rev- and CRM1-independent manner [13,14]. Nuclear pore complex proteins, such as nucleoporin p62 (Nup62), also gate HIV-1 vRNA for nucleocytoplasmic export [15]. While these examples identify positive activators of nucleocytoplasmic export, insulin-like growth factor II mRNA binding protein 1 (IMP1) and HS1-associated protein X-1 (Hax-1) are host factors that negatively regulate Rev function 
during nuclear export $[16,17]$. Thus, the mechanisms and virus-host interactions surrounding these events represent viable targets to block HIV-1 gene expression and assembly [18].

UPF1 is an RNA-binding protein that possesses RNA helicase and ATPase activities, Zn-coordinating finger motifs as well as acidic and basic amino acid clusters [19,20]. It shuttles between the nucleus and cytoplasm via CRM1 and this characteristic imparts potential roles in both the nucleus and cytoplasm [21]. The roles of UPF1 are quite diverse in mammalian cells as reviewed in [22] and include RNA stability [23,24], DNA repair, cell cycle progression [25], DNA replication and telomere metabolism [26]. UPF1 exerts these functions in the context of several nuclear and cytoplasmic ribonucleoprotein (RNP) complexes [24,26-31]. Its best understood and characterized function lies in its role in nonsense-mediated mRNA decay (NMD), an RNA surveillance mechanism that targets mRNAs that harbour premature termination codons (PTC) for degradation [30,32]. NMD has been shown to regulate the stability of many physiological transcripts, thus implying an important role as a post-transcriptional regulator of gene expression, as reviewed in $[19,33]$. For example, miR-128 is a brain-specific miRNA that targets, among others, the UPF1 transcript leading to the repression of NMD and the concomitant upregulation of many proteins controlling neuronal development and function in differentiating neural cells [34]. The process of NMD has been shown to be tightly self-regulated to ensure that its regulatory and surveillance functions are not disturbed. As such, most transcripts encoding NMD factors are sensitive to NMD themselves [35,36]. The proteins involved in NMD include the UPF proteins (UPF1, UPF2 and UPF3) that form a complex that interacts with the exon-junction complex (EJC) via UPF3b [37,38]. This model is evolving, since new evidence suggests that UPF1 initially binds mRNAs before aberrant translation termination triggers NMD [39,40]. In addition, a UPF2-independent NMD pathway has also been described, obviating the requirement for UPF2 [41].

In addition to transcripts carrying a PTC, substrates for NMD include, among others, mRNAs harbouring long 3'-untranslated regions (UTR), introns in the 3'-untranslated region (3'-UTR) and frameshift sequences $[33,39,42]$. However, retroviruses are able to evade recognition by the NMD RNA quality control machineries, despite possessing very long 3'-UTRs [43]. Indeed, HIV-1 makes use of UPF1 during viral replication to promote vRNA stability in both nuclear and cytoplasmic compartments as well as vRNA translation in the cytoplasm, directly influencing steady-state levels of the main viral structural protein, Gag, and thus, virus production [5]. Recent findings suggest that UPF1 could exert this activity in part by a rapid association with the RNA during its de novo vRNA synthesis, immediately following transcription [44], observations consistent with the results presented herein (see below). In the cytoplasm, UPF1 assembles in a complex in which the vRNA, the viral structural protein Gag and host proteins Staufen1 and UPF3b are present but from which UPF2 is excluded [5,45]. All of these components are found in complex with UPF1 but the assembly into the HIV-1 RNP could also be mediated via an interaction with the vRNA [5,44]. It was also recently shown that UPF1 associates with the HIV-1 RNA in an RNA length-dependent manner [44,46].

UPF2 is a phosphoprotein that interacts with UPF1 and UPF3b to trigger NMD. In at least one study, the interaction between UPF2 and UPF1 was shown to be mediated by a conformational change of UPF1 from an RNA-binding to an RNA unwinding mode dampening UPF1's ability to bind RNA [28]. The other component of the surveillance complex, UPF3, has two paralogs, UPF3a and UPF3b, and they both trigger NMD differently [47]. UPF3a has two isoforms, UPF3aL and an additional one called UPF3aS, which lacks exon 4 and binds UPF1 but not UPF2 [48]. Moreover, both UPF3aL and UPF3aS 
are found in different complexes: UPF3aS/PP2A/SMG5/SMG7 and phosphorylated UPF1 (P-UPF1) (called pre-dephosphorylation) and a complex lacking SMG5/7 but containing UPF2, UPF3aL and P-UPF1 (called post-phosphorylation) [31]. Moreover, UPF3b regulates UPF3aL protein levels, but this is dependent on their ability to associate to UPF2 [49]. This indicates that UPF3b and UPF3aL/S have differential roles in distinct RNPs. Moreover, P-UPF1 preferentially binds to both SMG5/7 and SMG6, and the knockdown of SMG6 results in an increase of UPF1 in complex with SMG5/7, thereby decreasing the abundance of UPF1 associated to UPF2-UPF3aL [31,50].

Since our earlier work strongly supported a nuclear role for UPF1 [5], in this report we characterize the importance of the nuclear interaction between UPF1 and the vRNA. We demonstrate that UPF1 shuttling promotes the nucleocytoplasmic export of vRNA. UPF1 expression also overcomes the nuclear retention of vRNA due to the absence of Rev expression. Importantly, using in situ imaging analyses and in silico modeling of protein-protein interactions, we revealed that the association between UPF1 and UPF2 plays a critical role in the regulation of vRNA nucleocytoplasmic export. These results concretely explain why HIV-1 excludes UPF2 from its nuclear export and cytoplasmic HIV-1 RNP but also identify an unsuspected regulatory circuit involving multiple host UPF proteins in determining the fate of the HIV-1 vRNA.

\section{Methods}

\subsection{Cell Culture, Plasmids and Transfections}

Cell culture of HeLa cells and transfections of proviral DNAs, pNL4-3 and pMRev(-) as well as UPF1 siRNA and rescue experiments were performed as described before [5]. Total cellular RNA was isolated by TriZol Reagent or TriZol LS (Life Technologies, Carlsbad, CA, USA) according to the manufacturer's instructions. pMRev(-) construct was provided by the NIH AIDS Reference and Reagent Program (generously provided by Reza Sadaie). pCl-FLAG-UPF1 (UPF1 ${ }^{\mathrm{WT}}$ ) and pCI-FLAG were described earlier [5]. Bryan Cullen (Duke University, Durham, NC, USA) provided the HA-TapA17 plasmid [51] and Alan Cochrane (University of Toronto, Toronto, ON, Canada) provided the CTE-Gag plasmid. The following GFP-tagged UPF1 wildtype (UPF1 ${ }^{\mathrm{WT}}$ ) and UPF1 mutants were described earlier [21]. Because the NES has been defined as rather large, the $\triangle$ NES mutant lacks the nuclear export signal (NES) as well as both zinc fingers and $\triangle$ NLS lacks the RNA helicase domains II and III as well as the nuclear localization signal (NLS) (as shown in Figure 1A). Rev-R-YC was provided by Ruth Brack-Werner (GSF-National Research Center for Environment and Health, Neuherberg, Germany) [52]. FLAG-UPF3aS, FLAG-UPF3aL, FLAG-UPF3b, FLAG-UPF2 ${ }^{\text {WT }}$, FLAG-UPF2 ${ }^{1173}$ and FLAG-UPF2 ${ }^{1-1096}$ were previously described $[38,48,49,53,54]$. FLAG-UPF3aS lacks exon4 from UPF3aL; FLAG-UPF2 ${ }^{1173}$ has point mutations which abolish its interaction with UPF1; FLAG-UPF2 ${ }^{1-1096}$ has a deletion at the C-terminus of the protein and does not bind UPF1 [47,48,53-55]. UPF proteins were overexpressed approximately 2-5-fold over western blotting signals obtained for endogenous UPF proteins. For the Leptomycin B (LMB) experiments, cells were transfected $20 \mathrm{~h}$ prior to collection and treated with $2 \mathrm{nM}$ of LMB (Sigma-Aldrich, Oakville, ON, Canada) as described previously [56]. Monocytic OM10.1 cells were mock induced or induced with phorbol ester PMA and cell lysates were harvested for western analyses at the indicated time points. siRNA experiments for siNS and siUPF1 were performed as previously described [5]. 


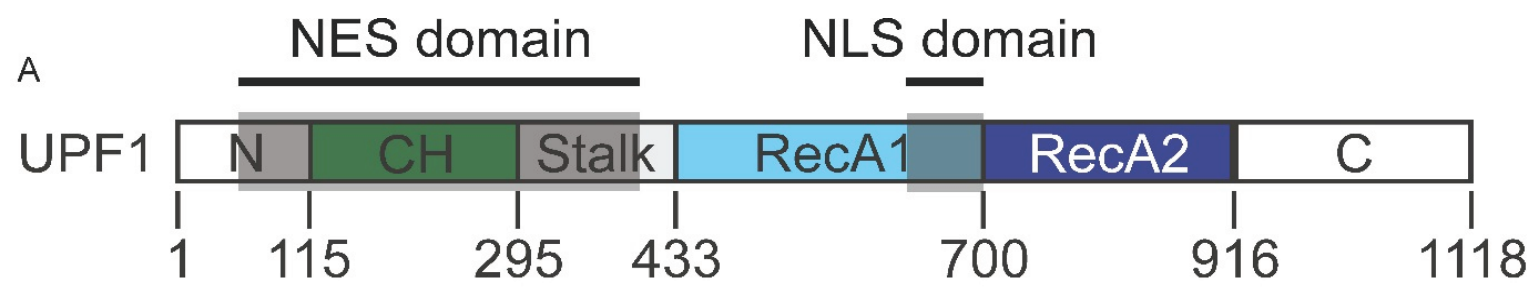

B

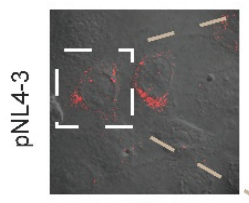

C

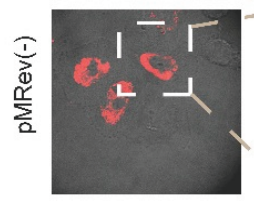

D

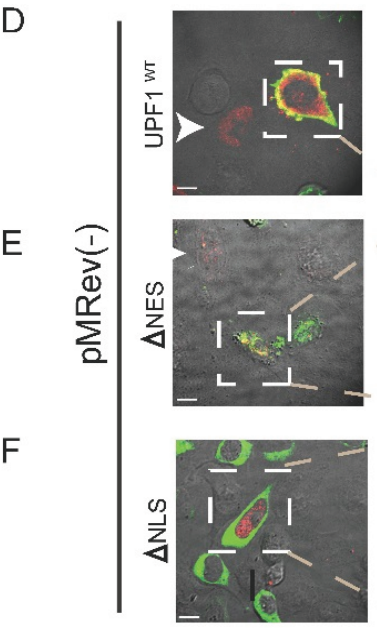

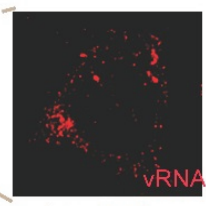
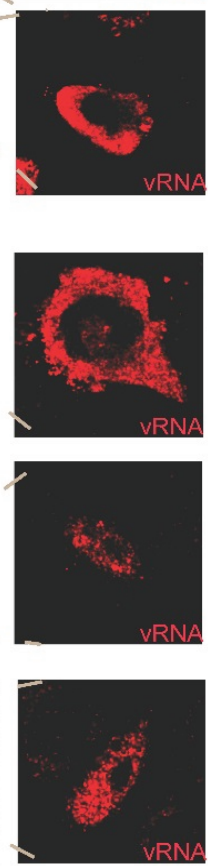
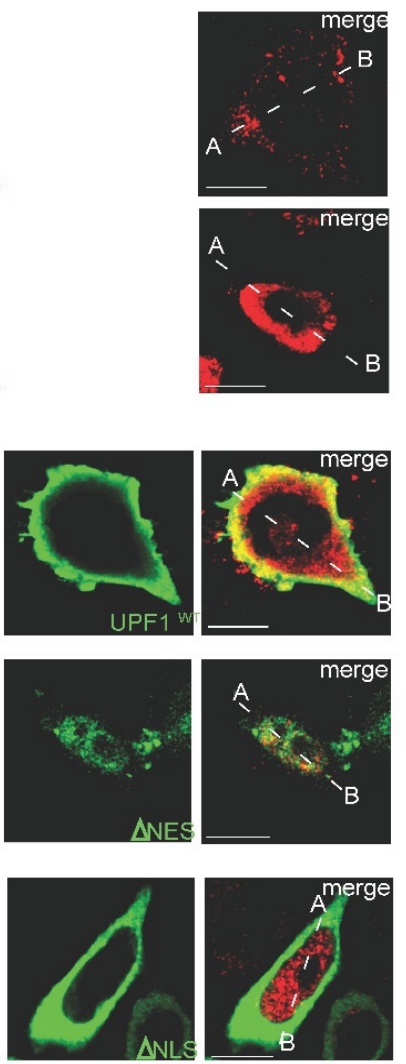

$$
\text { G }
$$

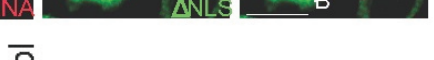

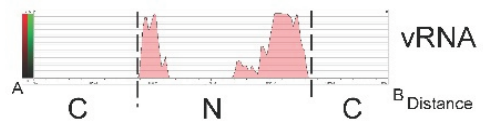

INTENSITY PLOTS
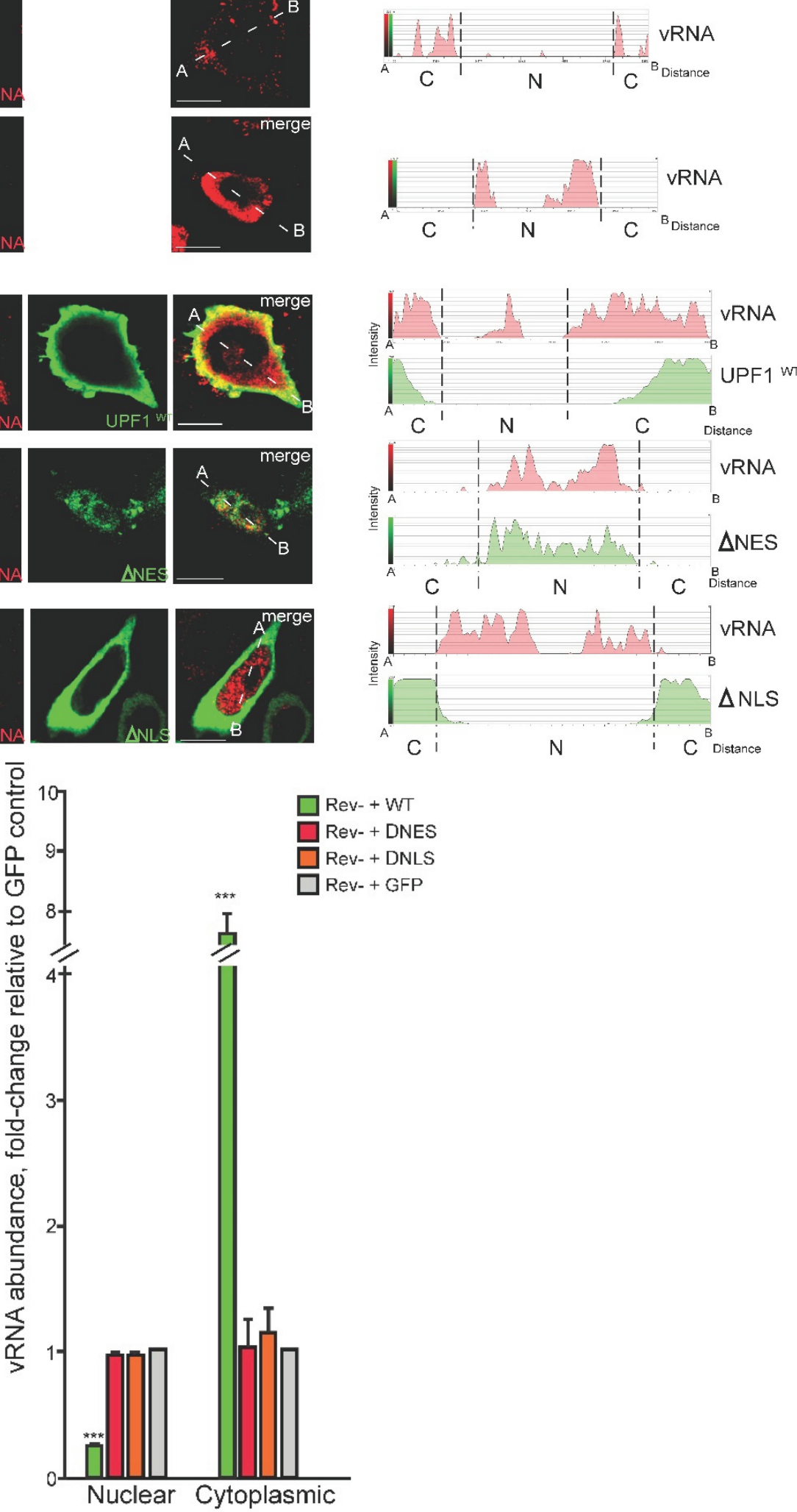
Figure 1. UPF1 nucleocytoplasmic shuttling promotes vRNA nucleocytoplasmic export (A); The UPF1 nuclear export and localization sequence (NES, NLS) domains are shaded in the depiction of UPF1. HeLa cells were co-transfected with wildtype proviral DNA (PNL4-3) alone (B); or a Rev-defective provirus pMRev(-) alone (C); or with $\mathrm{UPF} 1^{\mathrm{WT}}(\mathbf{D}) ; \mathrm{UPF}^{\Delta \mathrm{NES}}(\mathbf{E})$; and $U P F 1^{\triangle N L S}(\mathbf{F})$. vRNA shown in red and UPF1 proteins are shown in green (in all panels). White arrowheads identify HIV-1-transfected cells. Size bars indicate $10 \mu \mathrm{m}$. The corresponding Intensity plots for vRNA (red) and GFP-tagged UPF1 protein staining are shown from point A to $\mathrm{B}$ in the designate cells: dashed lines demarcate the nucleus $(\mathrm{N})$ and cytoplasm $(\mathrm{C})$ boundaries. Imaging results shown are representative of the phenotypes observed in $>85 \%$ cells in each condition in 5 independent experiments. (G) Dunnett post-test analyses on vRNA localization results \pm SEM values compared to the controls pNL4-3+GFP (pNL4-3) or GFP (Rev-); *** $p<0.001$.

\subsection{Antisera and Reagents}

Antisera to UPF1, UPF2 and UPF3b were generously supplied by Jens Lykke-Andersen (University of California, San Diego, CA, USA). Antisera to DDX3 was generously supplied by Luc DesGroseillers (University of Montreal, Montreal, PQ, Canada). Mouse anti-p24 and mouse anti-GAPDH antisera were purchased from Intracell and Techni-Science, respectively. Mouse anti-Digoxin was purchased from Sigma-Aldrich and sheep anti-Digoxin was purchased from Roche. Mouse and Rabbit anti-FLAG antibodies were purchased from Sigma-Aldrich and mouse anti-GFP was purchased from Roche. Rabbit anti-CRM1 was purchased from Santa Cruz. Rabbit and mouse anti-HA was purchased from Santa Cruz and Roche, respectively. The Nup62 antibody was purchased from Sigma-Aldrich and rat anti-RFP (identifying mCherry in IF analysis) was from Chromotek and the anti-SMG6 was from Abcam. For western analysis, horseradish peroxidase-conjugated anti-rabbit and anti-mouse antibodies were purchased from Rockland Immunochemicals (Pottstown, PA, USA). Signal intensities were scanned by densitometry using either Image J software (NIH, Bethesda, MD, USA) or using the Gel Doc System and quantitated by the Quantity One Software version 4.4.1 (BioRad, Montreal, QC, Canada) [57]. Standard curves were generated using cell extracts to ensure that signal intensities fell within the linear ranges of the assays using the antibodies described in the manuscript. For indirect IF and FISH, secondary fluorophore-conjugated antisera AlexaFluor donkey anti-mouse 488, 594 and 647, donkey anti-sheep 488 and 647 and donkey anti-rabbit 594 and 647 were purchased from Invitrogen [56].

\subsection{FISH/IF Co-Analyses}

Fluorescence in situ hybridization and immunofluorescence (FISH/IF) co-analyses were performed as described earlier [56,58-60]. The antisense RNA probe recognizes a 236bp long region in the pol gene (nt 1724-1960) and was prepared by in vitro transcription with digoxigenin-labeled UTP (Roche Applied Science, Montreal, QC, Canada). Microscopy was performed on a Zeiss LSM5 Pascal laser-scanning confocal microscope (LSCM) or on a WaveFX spinning disk confocal microscope system (Quorum Technologies, Inc., Guelph, ON, Canada). Microscopy techniques, filter sets and laser wavelengths were completely described earlier [56,58]. Quantitative imaging analyses were performed with the help of Imaris software v. 7.4 (Bitplane, Inc., Zurich, Switzerland). Intensity plots for antigen/vRNA signals was 
achieved using the measurement panel through the center focal plane, as described previously $[58,61]$. All imaging experiments were performed at least 3 and as many as 7 times with similar results. Nuclear vs. cytoplasmic phenotypes were defined only when $>80 \%$ of the vRNA was localized in the nucleus or in the cytoplasm. Representative phenotypes are shown in the figures in the manuscript. The observed phenotypes were obtained in $78 \%$ to $85 \%$ cells ( $n>50$ cells per condition) in each experiment. Data were analysed by one-way analysis of variance followed by a Dunnett post-test using GraphPad Prism 5.0 software. This test compares the mean of each condition with that determined for the respective control. The histograms shown in the manuscript represent the fold-change in vRNA localization $( \pm$ standard error of the mean, SEM) in either nuclear or cytoplasmic compartments with respect to control conditions. Quantitative evaluation of the localization was achieved by visual inspection of the central confocal plane of laser scanning confocal images; cells were excluded from the calculation when either the signal intensity for HIV-1 or UPF antigens was poorly detectable or when the distribution was not distinct such that it spanned the nucleus, nuclear periphery and cytoplasmic compartments. In most experiments, distinguishing the nucleus/cytoplasm limits was readily observable and in some experiments the use of the chromatin stain, DAPI or immunofluorescence of Nup62 was used to confirm the cytoplasm/nucleus boundary. Only cells in which HIV-1 and the co-expressed proteins were considered in the calculations, where applicable. A $p$ value of $<0.05$ was considered statistically significant. The RNA-specific dye SYTO14 was used to localize bulk mRNAs in the study as described earlier [62] and the expression of the reporter gene mCherry (CMV-mCherry [63]; kindly provided by Paul Bieniasz, Aaron Diamond AIDS Center, New York, NY, USA) followed by biochemical cell fractionation analysis was used to assess the effects of UPF protein overexpression on mCherry and mCherry mRNA localization, a representative unrelated mRNA (to HIV-1 RNA). Overexpression of UPF constructs had little effect on bulk mRNA localization (see Supplemental Figure S1).

HeLa cells were transfected as described above and were described earlier [5]. For the FLAG immunoprecipitation, $1 \mathrm{mg}$ of total lysate was incubated $2 \mathrm{~h}$ with agarose conjugated anti-FLAG beads and the bound complex was analyzed via SDS-PAGE analysis as described earlier [61]. For the Gag immunoprecipitation HeLa cells were transfected as described above. Cells were lysed in NP40 lysis buffer and Gag was immunoprecipitated as described previously using $1 \mathrm{mg}$ of protein and affinity-purified mouse anti-p24 antisera (hybridroma 183-H12-5C) [64] from the NIH AIDS Reference and Reagent Program [5]. Signal intensities in immunoprecipitates were normalized to input protein levels found in total cell lysates and were quantitated by densitometry as described above. The lack of GAPDH immunoreactivity in the immunoprecipitates (output) demonstrated the specificity of this assay.

\subsection{First STRAND cDNA Synthesis Followed by PCR}

HeLa cells were transfected as described above and were described earlier [5]. For cell fractionation studies, the nuclear and cytoplasmic fractions were prepared as previously described prior to western analysis [15,65] and performed at least twice for each type of experiment. RNA was purified using TriZol and Trizol LS. $1 \mu \mathrm{g}$ of total RNA was treated with DNAse I Amplification Grade (Life Technologies) and first strand cDNA synthesis was performed using Superscript II RT (Life Technologies). $2 \mu \mathrm{L}$ of total cDNA was taken and PCR amplified for gapdh mRNA and vRNA using the primers described in Abrahamyan et al. [61]. mCherry mRNA was amplified using a specific primer pair (Forward primer, 
5'-TGG AGG GCT CCG TGA ACG GCC and reverse primer 5'-TAG GCG CCG GGC AGC TGC ACG) to generate a RT-PCR product of about $483 \mathrm{bp}$. Signal intensities of PCR signals (cycle 25-30) were quantitated exactly as described above [61,62] using ethidium bromide stained DNA signals captured in-gel. All calculations were performed by taking into account the gapdh mRNA signal intensities.

\subsection{Glycerol Gradient Fractionation of UPF1 Complexes}

$500 \mu \mathrm{g}$ protein from lysates derived from cells expressing HIV-1 pNL4-3 and pCi-FLAG-UPF1 was immunoprecipitated using FLAG-M2 agarose beads (Sigma-Aldrich). Immunoprecipitates were eluted using FLAG-3X peptide as per the manufacturer's protocol. The eluted FLAG-UPF1 complexes were then carefully layered on a $5 \%-50 \%$ glycerol gradient and centrifuged at $80,000 \times g$ for $2 \mathrm{~h}, 4{ }^{\circ} \mathrm{C} .12-500 \mu \mathrm{L}$ fractions were collected and the abundance of FLAG-UPF1, pr55 ${ }^{\mathrm{Gag}}, \mathrm{CRM} 1$ and UPF3b were characterized by Western blotting. The vRNA was extracted using TriZol according to the manufacturer (Invitrogen, Montreal, QC, Canada) and quantitated by RNA slot blotting analyses, exactly as described [66].

\subsection{Protein-Protein Docking and Alanine Scanning}

Protein-protein docking was performed using the structure of UPF1 (PDB: 2WJV) and DDX3 (PDB: 2I4I), previously described [53,67]. To obtain the structure of a Rev, we used the structure of the L12S/L60R mutant Rev dimer (PDB: 3LPH) and the structure of a wild type Rev monomer (PDB: 2X7L), previously described $[68,69]$. For this, we proceed with structural analysis between the dimer and monomer using the SALIGN module of MODELLER, version 9.13 [70]. We performed the protein-protein docking using the ZDOCK web server (Pierce, B.G. et al., 2014) with default parameters and without specifying amino acids for interaction. Then, the models with the best score were refined using the Rosseta server [71]. For alanine scanning, the Rossetta server was used and amino acids with $\Delta \Delta \mathrm{G} \geq 1 \mathrm{kcal} / \mathrm{mol}$ were defined as relevant for interaction as suggested [72]. Drawing of structures was performed with Pymol (The PyMOL Molecular Graphics System, Version 1.4.1 Schrödinger, LLC).

\section{Results}

\subsection{UPF1 Shuttling Is Important for $v R N A$ Nuclear Export}

Our earlier work showed that the expression of either the wild-type UPF1 (UPF1 ${ }^{\mathrm{WT}}$ ) or the trans-dominant negative (TDN) NMD-null RNA helicase mutant, UPF1 ${ }^{\text {R844C }}$, increased HIV-1 Gag and vRNA levels, indicating that UPF1's function in vRNA metabolism is independent of its role in NMD [5]. While NMD does require an interaction between UPF1 and UPF2 in most circumstances [53], this association is not essential for the resulting effects on HIV-1 gene expression as UPF2 is excluded from a cytoplasmic Gag RNP [5] and UPF1 mutants that do not interact with UPF2 maintain Gag and vRNA upregulation. The effects of UPF1 are also exerted in the nuclear compartment, such that UPF1 depletion decreased steady-state levels of nuclear HIV-1 vRNA, but had little effect on the spliced RNA species, under Rev- conditions when vRNA is retained in the nucleus [5]. UPF1 overexpression in these conditions resulted in slight increases in Gag expression but more marked increases in vRNA (similar to that found in Rev+ conditions) (Supplemental Figure S2; [5]), suggesting that UPF1 promotes vRNA nuclear export in the absence of Rev but Rev expression appears to be important for Gag upregulation, 
as we have shown previously [62]. To determine the magnitude of the effect of UPF1 in the nucleocytoplasmic shuttling of the vRNA, we tested whether UPF1 could impact HIV-1 vRNA localization under Rev- conditions. We co-expressed GFP-tagged UPF ${ }^{\text {WT }}$ and two shuttling mutants, one lacking a nuclear export signal $(\triangle \mathrm{NES})$ and one lacking a nuclear localization signal ( $\triangle \mathrm{NLS}$; depicted in Figure 1A) with proviral DNA pMRev- and examined HIV-1 vRNA localization. In this manuscript, vRNA is detected by sensitive fluorescence in situ hybridization/immunofluorescence (FISH/IF) co-analyses coupled with laser scanning confocal microscopy (LSCM) [59,60]. As expected, the expression of the Rev-deficient provirus $\operatorname{pMRev}(-)$ prevented vRNA export as most of the signal was observed in the cell nucleus (compare Figure 1B,C; and accompanying intensity plots). Surprisingly, the ectopic expression of UPF ${ }^{\mathrm{WT}}$ resulted in the translocation of the vRNA to the cytoplasm as evidenced by the prominent cytoplasmic signal of the vRNA (Figure 1D). In sharp contrast, the expression of the shuttling mutants of UPF1, UPF $1^{\triangle \mathrm{NES}}$ and UPF $1^{\triangle \mathrm{NLS}}$, did not promote vRNA nuclear export (Figure 1E,F). The relocalization of the HIV-1 vRNA under Rev- and UPF1 overexpression conditions correlates with the increase in Gag expression observed (Supplemental Figure S2) and was corroborated in subcellular fractionation assays (Supplemental Figure S1). This ectopic expression of UPF1 may impact on the genomic RNA at several levels (see Discussion).

The effect of UPF1 on vRNA localization was specific as we did not observe any major changes in total mRNA distribution upon expression of UPF1 as determined by staining with the dye, SYTO14 (Supplemental Figure S3A). Moreover, UPF1 overexpression did not affect the localization of mRNA and protein levels of the unrelated mCherry (Supplemental Figure S3B (compare lanes 5 and 8 with 6 and 9, respectively) Statistical Dunnett post-test analyses on vRNA localization patterns compared to control levels (Rev- and GFP control vector) showed that the overexpression of UPF ${ }^{\mathrm{WT}}$ promoted a significant translocation of vRNA to the cytoplasm in Rev- conditions (Figure 1G). Although the expression of $\mathrm{UPF} 1^{\triangle \mathrm{NES}}$ had little effect on vRNA localization (Figure 1E and corroborated in subcellular fractionation experiments, Supplemental Figure S1), this mutant UPF1 maintained an interaction with the vRNA, whereas UPF $1^{\triangle N L S}$ did not, indicating that the vRNA:UPF1 interaction likely initially occurs in the nuclear compartment [73]. These results demonstrate that the shuttling activity of UPF1 is important for directing HIV-1 vRNA to the cytoplasmic compartment. The characteristics and binding partners of $\mathrm{UPF}^{\mathrm{WT}}{ }^{\mathrm{WT}}, \mathrm{UPF} 1^{\triangle \mathrm{NES}}$ and UPF1 ${ }^{\triangle \mathrm{NLS}}$ as well as a summary of their effects on Rev- HIV-1 vRNA export are summarized in Table 1.

In earlier work, we showed that the abundance of vRNA correlated directly with the UPF1 expression levels in both Rev+ and Rev- conditions [5] supporting a role in vRNA stability. In addition, rescuing UPF1 following siRNA-mediated depletion led to a complete recovery of HIV-1 Gag synthesis but vRNA was not completely rescued, demonstrating that UPF1 also acted on vRNA translation [5]. Here, we determined how UPF1 depletion influenced the localization of HIV-1 vRNA. To do this, we depleted UPF1 and then rescued expression and performed subcellular fractionation and identifed nuclear and cytoplasmic marker proteins. Upon UPF1 depletion, vRNA markedly partitioned to the nuclear compartment with a concomitant 3-fold decrease in abundance in cytoplasmic levels (Figure 2A). We then performed a partial knockdown of UPF1, since the depletion of UPF1 usually leads to below-threshold detection of vRNA [5]. In cells with detectable vRNA signals, FISH/IF co-analyses showed that HIV-1 vRNA accumulated or was retained in the nucleus upon UPF1 depletion in $>80 \%$ cells $(n=48$; Figure $2 \mathrm{~B})$. This was a rarely captured observation indeed, because vRNA levels are highly sensitive to steady-state 
UPF1 expression levels [5]. These data indicate that a deficiency in UPF1 leads to the nuclear retention of vRNA.
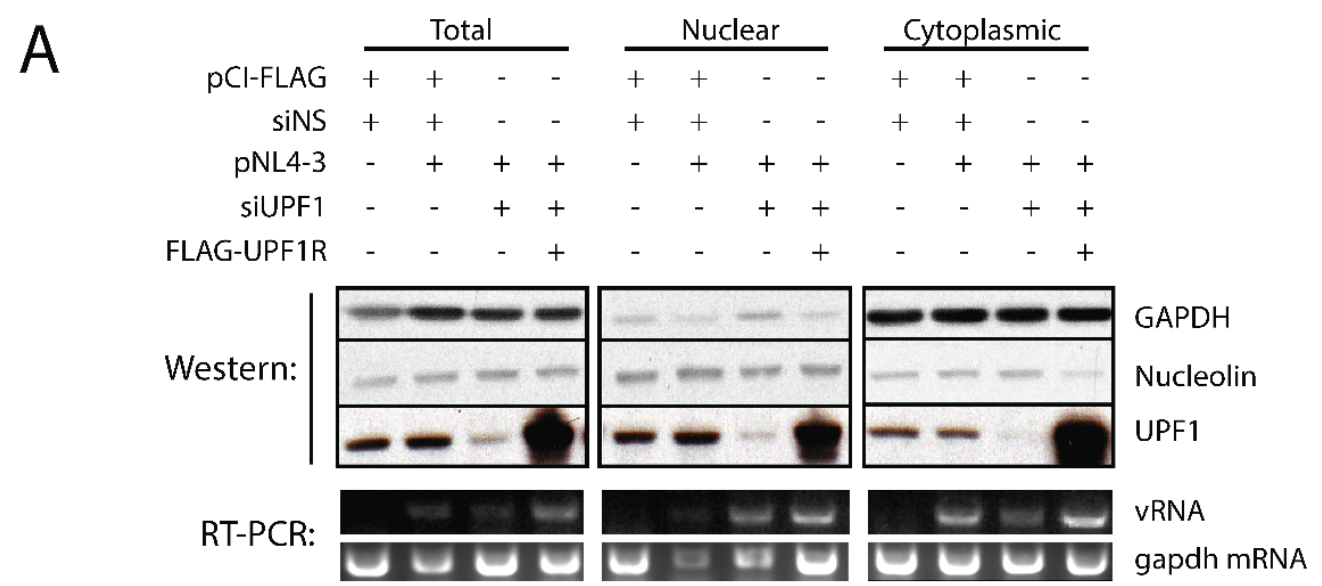

\%vRNA compared to siNS control: nd 10047114 nd 100146114 nd 10035137

B

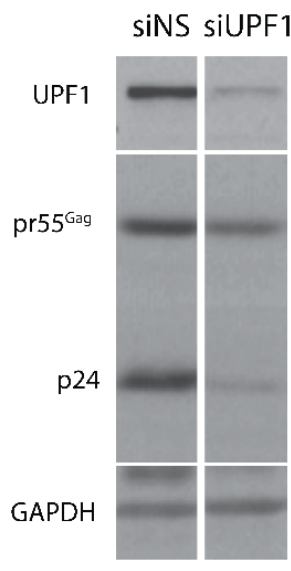

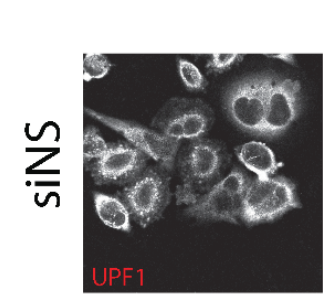

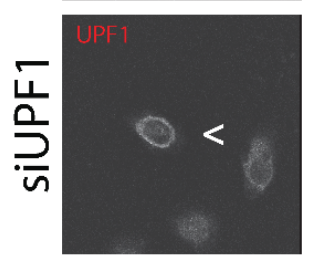

pNL4-3

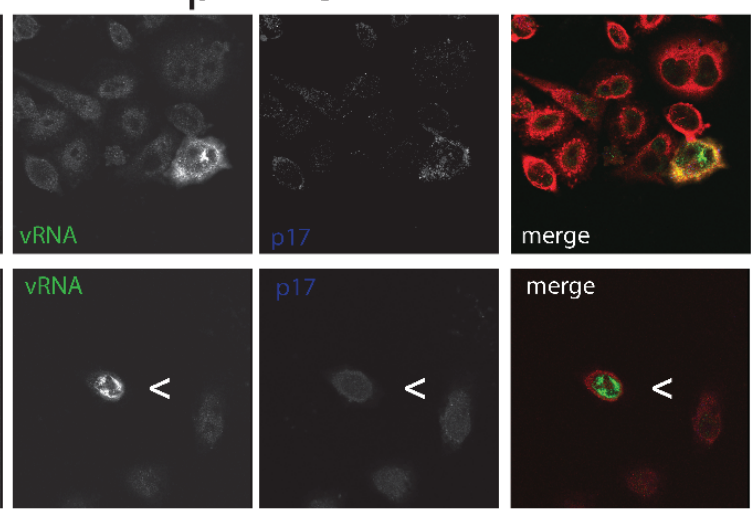

Figure 2. UPF1 depletion increases the abundance of vRNA in the nucleus while UPF1 Rescue enhances cyto-plasmic vRNA levels. (A) HeLa cells were mock transfected with pCI-FLAG and non-silencing siRNA (siNS) or co-transfected with HIV-1 proviral pNL4-3 DNA and pCI-FLAG and siNS. UPF1 was depleted by siUPF1 and UPF1 expression was rescued with FLAG-UPF1Rescue (UPF1R). At 30h post-transfection, cells were harvested and cytoplasmic-nuclear fractionation analysis was carried out as described in Materials and Methods. Western blot analysis was performed GAPDH (enriched in the cytoplasmic), Nucleolin (enriched in the nucleus), and UPF1 (identifying endogenous and overexpressed proteins). RNA was isolated from cell extracts and steady-state levels of vRNA and gapdh mRNA was measured by RT-PCR. vRNA levels were quantified by densitometry with ImageJ using gapdh mRNA signal intensity for normalization; pNL4-3 + siNS condition was set at a $100 \%$. nd, not detectable. (B) Cells were mock-depleted with nonsilencing siRNA (siNS) or depleted using siUPF1 as described in Materials and Methods. At 40-48 h post-transfection, cells were harvested for western blotting for UPF1 (endogenous), Gag (identifying pr55Gag and p24) and GAPDH (loading control) (on left) and FISH/IF co-analyses for UPF1 (red), vRNA (green) and Gag (blue) (with merged renditions on right). UPF1 depletion led to decreased Gag expression as shown earlier (left panel; Ajamian et al., 2008) but also led to a blockade to vRNA nuclear export (white arrowhead $<$ in lower row of right panel) in $>80 \%$ cells examined $(n=48)$. See results for additional discussion of these findings. 
Table 1. UPF1 plasmids cited in this report.

\begin{tabular}{|c|c|c|c|c|c|c|}
\hline \multirow[b]{2}{*}{$\begin{array}{l}\text { UPF1 } \\
\text { Plasmids }\end{array}$} & \multirow[b]{2}{*}{$\begin{array}{l}\text { Deleted } \\
\text { Region }\end{array}$} & \multirow[b]{2}{*}{$\begin{array}{l}\text { Protein } \\
\text { Localization }\end{array}$} & \multirow[b]{2}{*}{ Characteristics } & \multirow{2}{*}{$\begin{array}{l}\text { Effect on HIV-1 } \\
\text { RNA Export } \\
\text { (Rev- Conditions) } \\
\end{array}$} & \multicolumn{2}{|c|}{ Interactions } \\
\hline & & & & & $\begin{array}{l}\text { UPF } \\
2\end{array}$ & Staufen 1 \\
\hline $\mathrm{UPF} 1^{\mathrm{WT}}$ & $\mathrm{NA}^{*}$ & Shuttling [21] & $\begin{array}{l}\text { Binds UPF3a [48]; UPF3b [48]; } \\
\text { Staufen1 [24,74]; UPF2 [48,74]; } \\
\text { Nup100/116 (hNup98) [75]; nuclear HIV-1 } \\
\text { vRNA [44]; and found in HIV-1 [61] and } \\
\text { SupT1 and THP1 HIV-1 cores [76] }\end{array}$ & $\begin{array}{l}\text { Positively affects } \\
\text { export under } \\
\text { Rev- conditions [5]; } \\
\text { (this study) }\end{array}$ & Yes & Yes \\
\hline UPF1 $1^{\triangle N E S}$ & $55-416$ & Nuclear [21] & $\begin{array}{l}\text { Lacks nuclear export signal domain, zinc } \\
\text { fingers }(\mathrm{CH} \text { domain }(115-295) \text { [21,77]; } \\
\text { required for UPF2 and Staufen1 binding [74] }\end{array}$ & $\begin{array}{l}\text { No effect under } \\
\text { Rev- conditions } \\
\text { (this study) }\end{array}$ & No & No \\
\hline UPF1 $1^{\triangle N L S}$ & $596-697$ & Cytoplasmic [21] & $\begin{array}{l}\text { Lacks nuclear localization signal domain, } \\
\text { helicase domains II and III }[21,77]\end{array}$ & $\begin{array}{l}\text { No effect on } \\
\text { Rev- export } \\
\text { (this study) }\end{array}$ & Yes & Yes \\
\hline
\end{tabular}

* NA: not applicable.

\subsection{The CRM1-Mediated Export Pathway Is Favoured by UPF1 for Efficient vRNA Export}

The two essential nucleocytoplasmic export pathways are those mediated by CRM1 and NXF1. HIV-1 uses both export pathways for the export of its different RNA transcripts such that multiply-spliced RNAs are constitutively exported via NXF1 while Rev-dependent transcripts including the vRNA, are exported in a regulated manner to the cytoplasm via CRM1 [9]. To differentiate between the use of these two export pathways mediated by UPF1, FISH/IF co-analyses were employed to visualize the localization of the vRNA under a pharmacologic block of CRM1 mediated nuclear export using Leptomycin B (LMB) or a block of NXF1 mediated nuclear export imposed by the expression of a negative transdominant NXF1 (HA-TapA17) [51]. In contrast to the typical cytoplasmic localization of vRNA from the wild type provirus (Figure 1B), LMB treatment blocked Rev-mediated nucleocytoplasmic export resulting in the accumulation of vRNA in the nucleus (Figure 3A, shown in green). LMB also blocked CRM1-mediated shuttling of endogenous and, in part, exogenously-expressed FLAG-tagged UPF1 that lead to a partial accumulation in the nucleus in several experiments ((Figure 3A,B; UPF1 is shown in red in this figure) as previously shown [21]). In contrast, a normal cytoplasmic and punctate distribution of the vRNA was observed when NXF1-mediated export was blocked using HA-TapA17 in Rev + conditions and with FLAG-UPF $1^{\text {WT }}$ overexpression (Figure 3C,D). UPF1 also exhibited a normal cytoplasmic localization at steady-state upon expression of HA-TapA17 (Figure 3D). Since the vRNA as well as a fraction of UPF1 was trapped in the nucleus when CRM1-mediated export was blocked, but not when NXF1-mediated export was blocked, we conclude that UPF1 promotes vRNA nucleocytoplasmic export primarily via the CRM1 export pathway.

To validate specificity, we determined if this newly characterized export function of UPF1, which is CRM1-dependent in the context of bona fide HIV-1 expression, is also required for CTE-directed export of gag mRNA. CTE is the constitutive transport element which serves as a nuclear export signal for unspliced viral RNA of type D retroviruses by binding to the cellular mRNA export factor NXF1. Therefore, we co-expressed Gag-CTE with either FLAG-UPF1 ${ }^{\text {WT }}$ or HA-TapA17 and assessed Gag expression levels by western blotting (Figure 3E). The overexpression of HA-TapA17 blocked NXF1-mediated export by $80 \%-90 \%$ and resulted in a decrease in Gag-CTE expression, while importantly, there was no change to Gag-CTE levels when FLAG-UPF1 ${ }^{\text {WT }}$ was expressed (Figure 3E). These results 
show that while UPF1 can promote the export of HIV-1 vRNA destined for CRM1 export, it cannot enhance CTE/NXF1-mediated export, at least in the context of Gag.

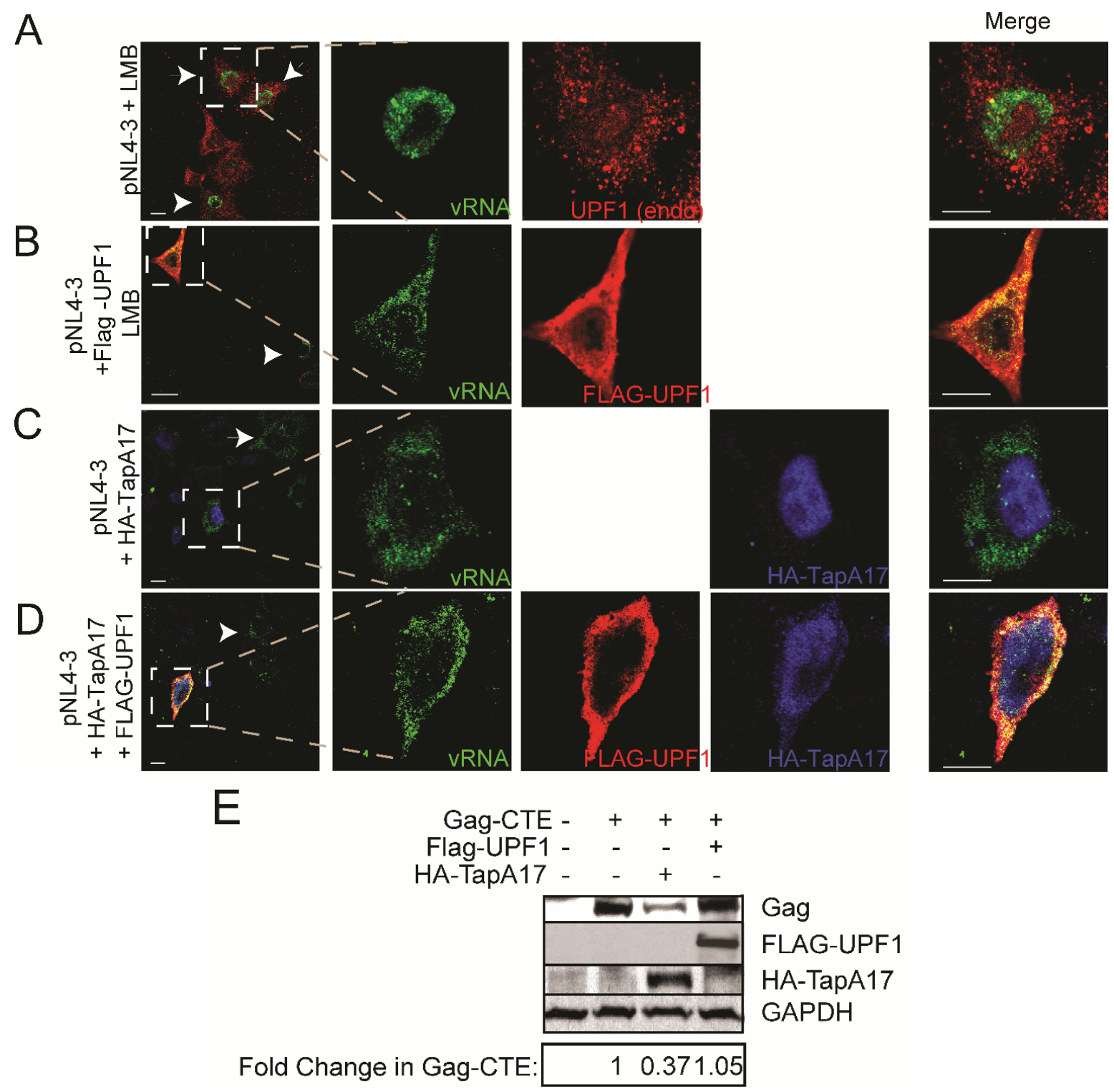

Figure 3. UPF1 exports vRNA via CRM1 and not the NXF1 export pathway. HeLa cells were transfected with pNL4-3 alone and treated with LMB (A); co-transfected with Flag-UPF1 and treated with LMB (B); co-transfected with HA-TapA17 (C); or co-transfected with HA-TapA17 and FLAG-UPF1 (D). FISH/IF co-analyses to identify the localization of vRNA (green in all rows), either endogenous UPF1 (red, A) or FLAG-UPF1 expressed in trans (red, $\mathrm{B}$ and D) or HA-TapA17 (blue in C and D). Merged renditions are shown on right-most panels. White arrowheads identify cells exhibiting LMB-induced block to nuclear vRNA export in (in A and B) and cells expressing HIV-1 (in C and D). Imaging results are representative of the phenotypes observed in $78 \%-85 \%$ cells in each condition in 3 experiments. Size bars, $10 \mu \mathrm{m}$; (E) Western blot analysis for Gag, FLAG-UPF1, HA-TapA17 and GAPDH (loading control). ImageJ was used to quantify the relative expression levels of Gag (normalized to GAPDH levels). Results shown represent averages from 2 independent experiments with $<10 \%$ deviation between experiments. nd: not detectable. 
HIV-1 encodes the regulatory viral protein Rev to export singly-spliced RNAs and the unspliced, vRNA to the cytoplasm [9]. Rev binds to the vRNA via an interaction between its NLS domain and the RRE element of the vRNA directing it through the nuclear pore via an interaction with CRM1 and nucleoporins $[10,78,79]$. Because our results highlight the importance of the shuttling function of UPF1 in vRNA export in both Rev-dependent and -independent conditions, we attempted to identify if UPF1 was present in the RNP complex required for vRNA nucleocytoplasmic export. To do this, we overexpressed FLAG-UPF1 ${ }^{\text {WT }}$ with or without a Rev expressor (Rev-R-YC) and immunoprecipitated FLAG-UPF1 ${ }^{\text {WT }}$. Rev, DDX3 and CRM1 all co-immunoprecipitated with UPF1 ${ }^{\text {WT }}$ in the presence and absence of Rev (Figure 4B). In earlier published work, we showed that the depletion of Nup62 resulted in a block to vRNA nuclear export and Nup62 was also found to be part of the HIV-1 Rev RNP complex [10,15]. We therefore sought to determine if UPF1 could also co-precipitate with Nup62. Indeed, Nup62 was also found to be part of the UPF1 RNP strengthening the role of UPF1 in vRNA export (Figure 4C). In order to reinforce the notion that UPF1 is involved in vRNA export and is present in two different complexes, we also performed glycerol gradient analysis on immunoprecipitated UPF1 complexes in HIV-1 positive conditions and found that in lighter fractions UPF1 is associated with CRM1 and the HIV-1 vRNA and in the more dense fractions it is associated with CRM1, UPF3b, Gag and vRNA, similar to the Staufen1HIV-1 RNP complex [5,45,80] (Figure 4D). In addition, DDX3, CRM1 and Nup62 were all absent from the HIV-1 Gag RNP complex as determined by co-IP analysis using anti-p24 [26] (Figure 4E) suggesting that UPF1 is found in at least two different HIV-1 RNPs: the first being composed of the vRNA and nucleocytoplasmic export factors such as CRM1, Rev, Nup62 and DDX3, most likely a nuclear RNP complex, and a second UPF1 cytoplasmic complex that includes Gag and a variety of other cytoplasmic proteins likely to be important for viral assembly, as we have proposed [5].

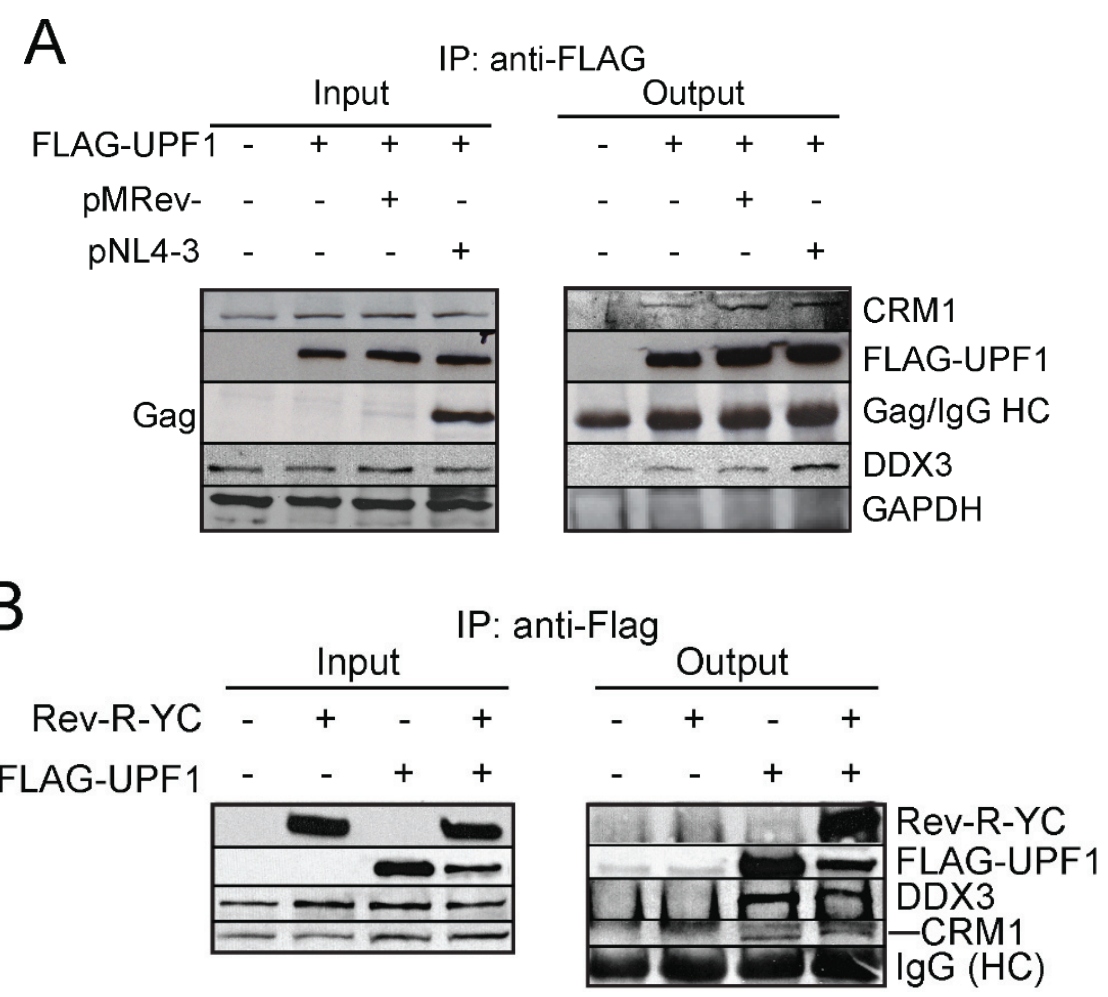

Figure 4. Cont. 


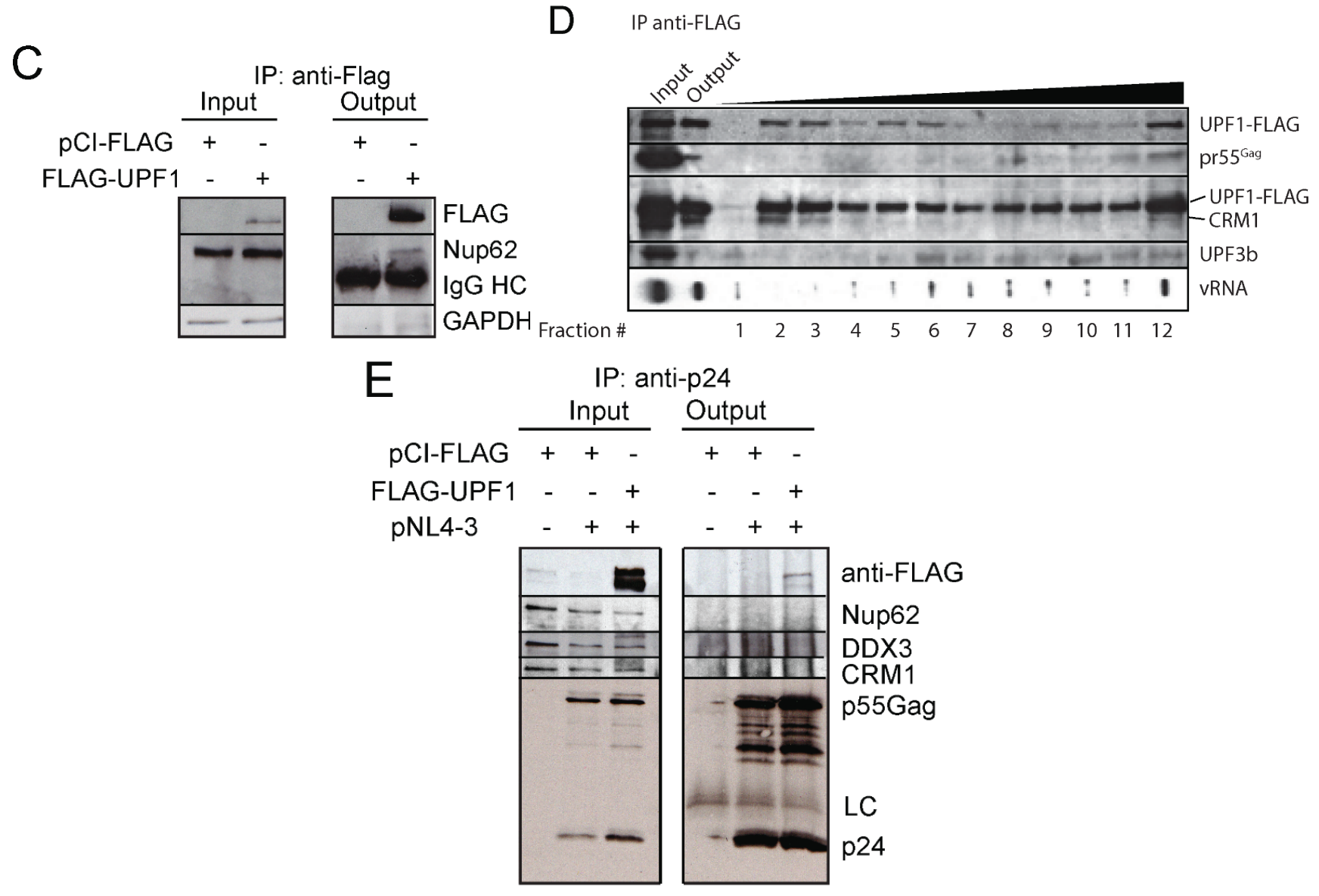

Figure 4. UPF1 is found in complex with Rev-CRM1-DDX3-Nup62. (A) HeLa cells were mock transfected with pCI-FLAG or transfected with FLAG-UPF1 alone or with either pMRev- or Rev+ pNL4-3 DNAs. FLAG-UPF1 was immunoprecipitated as described in Materials and Methods. Input lysates and bound complexes were analyzed by western blotting for CRM1, FLAG-UPF1, Gag, DDX3 and GAPDH (loading and output control). HC: Heavy Chain IgG. Results shown are representative of three independent experiments; (B) HeLa cells were mock transfected with pCI-FLAG or FLAG-UPF1 or co-transfected with FLAG-UPF1 and Rev-R-YC. FLAG-UPF1 was immunoprecipitated and western blotting of input lysates and bound complexes for Rev, FLAG-UPF1, DDX3 and CRM1. HC: Heavy Chain IgG; (C) HeLa cells were mock transfected with pCI-FLAG or transfected with FLAG-UPF1. Cells were harvested and FLAG-UPF1 was immunoprecipitated as described in Materials and Methods. Western blotting analysis for FLAG-UPF1 and Nup62. IgG HC: IgG heavy Chain; (D) Glycerol gradient analyses of UPF1 complexes in HIV-1-expressing cells. HIV-1/FLAG-UPF1-expressing cells were immunoprecipitated with anti-FLAG serum. FLAG-UPF1 complexes were eluted and fractionated on a glycerol gradient. Fractions were collected and FLAG-UPF1, pr55 $5^{\mathrm{Gag}}$, CRM1 and UPF3b were probed in each fraction by Western blotting. vRNA abundance is shown on the bottom panel, as determined by slot blot analyses; (E) HeLa cells were mock transfected with pCI-FLAG or co-transfected with HIV-1 and FLAG-UPF1. At $30 \mathrm{~h}$ post--transfection, Gag was immunoprecipitated using monoclonal anti-p24 antisera. Western for UPF1 (FLAG), Gag, CRM1, Nup62 and DDX3 were performed. Light Chain IgG. 


\subsection{A Model for the Assembly of the UPF1/DDX3/Rev Complex}

The results presented above show the formation of a multimeric complex containing UPF1, DDX3, CRM1 and Rev is necessary for nuclear export of the HIV-1 vRNA. UPF1 might bind to CRM1 through its NES as UPF1 ${ }^{\triangle N E S}$ is retained in the cell nucleus. On the other hand, DDX3 and Rev also bind directly to CRM1 through the helicase core domain and a NES, respectively [12,81]. However, details on the UPF1-DDX3 or the UPF1-Rev interaction are unknown. Thus, in order to characterize the interactions inside this particular RNP complex assembled during HIV-1 replication, we generate computational models through protein-protein docking (Figure 5). For this, we took advantage of previously described structures of UPF1 in the context of the UPF1-UPF2 complex [53] and of DDX3 in complex with AMP-PNP [67]. In order to obtain the structure of a wild type Rev dimer, we used the structure of the wild type monomer [68] and the structure of the dimer containing the L12S and L60R mutations [69]. It should be mentioned that the structure of the Rev dimer used for modeling lacks the NES and is not bound to the RRE. However, the NES was predicted to be projected away from the arginine-rich motif (ARM) and thus, may not interfere with protein folding [69]. Structural models for UPF1-DDX3 (Figure 5A) and UPF1-Rev (Figure 5B) were generated using the ZDOCK web server [82] and the models with the best score were refined with the ROSSETA server [71] as described in Materials and Methods.

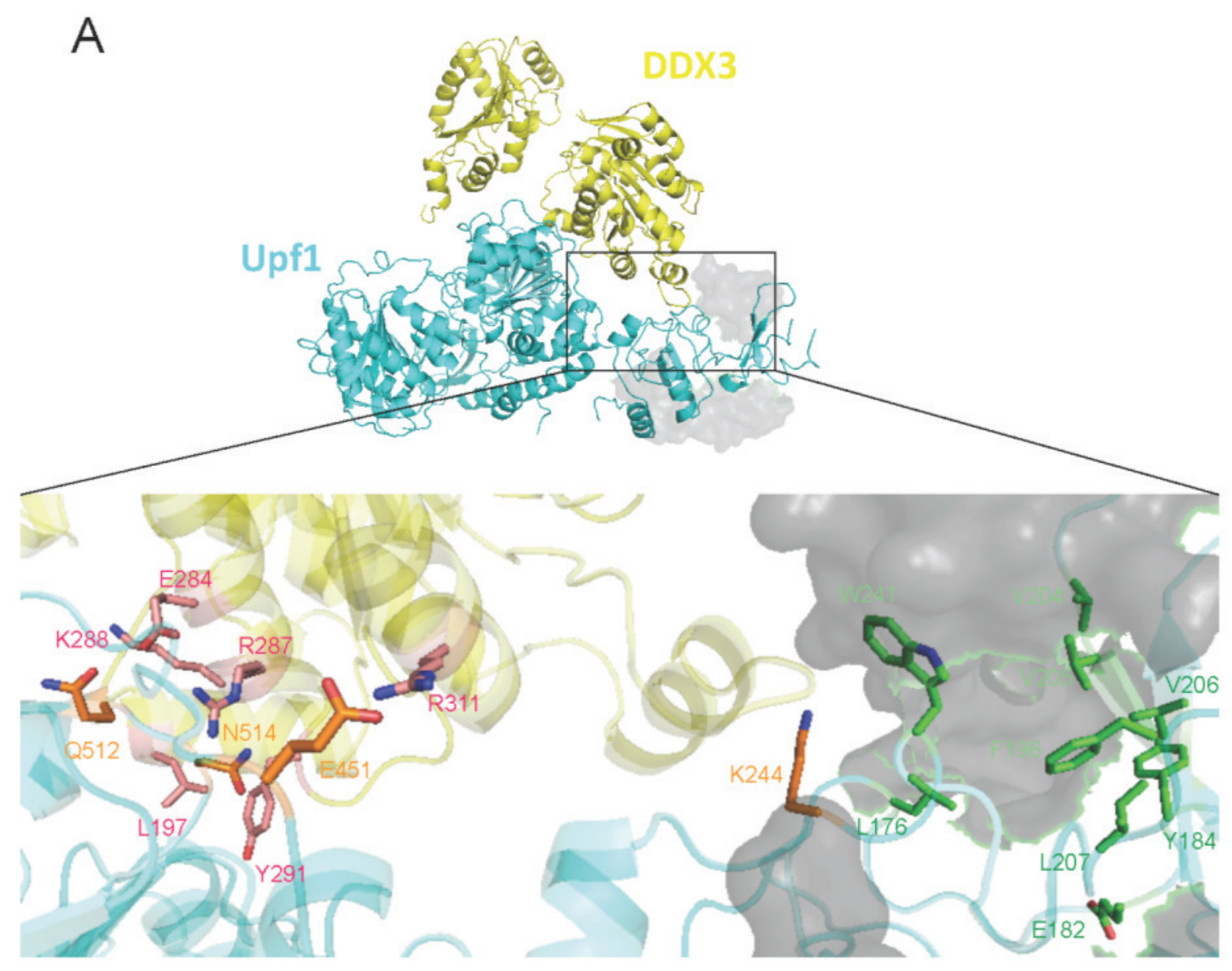

Figure 5. Cont. 




Figure 5. Interaction models and a mechanism for UPF2 exclusion. (A) Structural model for the interaction between UPF1 (cyan) and DDX3 (yellow) and structural alignment with the UPF1-UPF2 complex. UPF2 is represented as a gray surface (upper). An amplified image of the model for the UPF1-DDX3 complex is presented (bottom). Relevant residues obtained for UPF1 (orange) and DDX3 (pink) through alanine scanning or described in previous work as relevant for interaction with UPF2 (green) are shown as sticks. (B) Structural model for the interaction between UPF1 (cyan) and Rev (magenta) and structural alignment with the UPF1-UPF2 complex. UPF2 is represented as a gray surface (upper). An amplified image of the model for the UPF1-Rev complex is presented (bottom). Relevant residues obtained for UPF1 (orange) and Rev (pink) through alanine scanning or described in previous work as relevant for interaction with UPF2 (green) are shown as sticks. Drawing was performed with the Pymol software as described in materials and methods.

In the UPF1-DDX3 complex, we observed a wide surface of contact between the RecA-like domain 1 of DDX3 and the RecA-like domain 1A and the CH-domain of UPF1 (Figure 5A, upper panel). A structural alignment between the modeled structure of the UPF1-DDX3 complex and the structure of the UPF1-UPF2 complex [53] indicates that there is no apparent interference between DDX3 and UPF2 for 
binding UPF1 (Figure 5A, upper panel). Then, to understand the interaction between UPF1 and DDX3 at the molecular level, we perform an in silico alanine scanning using the Rosetta server as described in materials and methods [72]. We identified residues in the UPF1 Rec-like domain 1A domain (E451, N514 and Q512) and CH-domain (K244) as relevant for the interaction with DDX3 (Figure 4A, bottom panel). We also identified residues L197, E284, R287, K288, Y291 and R311 in the Rec-like domain 1 of DDX3 as relevant for the interaction with UPF1 (Figure 5A, bottom panel). Residues in UPF1 previously described as critical for its interaction with UPF2 [53,83] are shown in green (Figure 5A, bottom panel). Of note, our alanine scanning also identified UPF1 residue W241, a residue involved in UPF2 binding $[53,83]$ as relevant for interaction with DDX3 suggesting a partial overlap between DDX3 and UPF2 that could interfere with binding of the latter (Figure 5A, bottom panel).

In the Rev-UPF1 model, we observed that one of the monomers of Rev interacts with the CH-domain of UPF1, a region described as critical for interaction with UPF2 [53,83]. Indeed, our structural alignment revealed that Rev could bind UPF1 at the same region that binds the $\beta$-strand of UPF2 (Figure 5B, upper panel). Such a secondary structure in UPF2 was described as the most relevant for its interaction with UPF1 as mutations in the $\beta$-strand results in the disassembly of the UPF1-UPF2 complex $[53,83]$. Interestingly, two residues in UPF1 important for its interaction with UPF2 (W241 and L176) were identified by alanine scanning as relevant for Rev binding (Figure 5B, bottom panel). Moreover, our model also shows that additional residues in UPF1 that are relevant for its interaction with UPF2 such as F196, V204 and V05 could be completely masked by Rev (Figure 5B, bottom panel). Despite the limitations of our modeling analyses, it appears that in the context of HIV-1 infection, Rev could interfere with the formation of an UPF1-UPF2 complex, without interfering with the UPF1-DDX3 interaction. This nicely correlates with our previous work indicating that UPF2 is not required for the UPF1-mediated stimulation of HIV-1 expression and provides molecular insights into the how UPF2 is excluded from the UPF1 complex assembled during HIV-1 replication [5,45] (and see below). Nevertheless, biochemical analyses using mutants are required to validate the residues that are important for the UPF1-DDX3 and UPF1-Rev interaction.

\subsection{HIV-1 Induces the Exclusion of UPF2 from UPF1 Complexes}

The UPF1-3 proteins are components of different RNPs. For example, UPF1 exists in either UPF2-containing or UPF2-free NMD complexes. P-UPF1 is in complex with either UPF3aS/PP2A/ SMG5/SMG7 or UPF3aL/UPF2 [31]. We therefore wanted to determine if there was a change in the association between UPF1, UPF2 and UPF3b in HIV-1 expressing cells, especially since our earlier work demonstrated that UPF2 was excluded from the HIV-1 RNP and we identified UPF3b in this RNP even though its overexpression did not increase HIV-1 vRNA levels [5]. Thus, HeLa cells were transfected with or without proviral DNA pNL4-3 and with UPF1 ${ }^{\text {WT }}$. Gag was immunoprecipitated and the presence of UPF1, UPF2 and UPF3b was assessed in the immunoprecipitate (Figure 6A). The results show that UPF1 co-immunoprecipitated with Gag as previously reported, while UPF2 was absent from the Gag RNP (Figure 6A). UPF3b was also (weakly) present in the Gag RNP complex (Figure 6A). These results are consistent with our previous observations that showed that both UPF1 and UPF3b were present in the Staufen1-HIV-1 RNP complex, while UPF2 was absent [5,45]. We then determined if HIV-1 modulated the interactions of UPF proteins. HeLa cells were transfected with FLAG DNA (empty vector pCI-FLAG) 
or wild type UPF1 (FLAG-UPF1 ${ }^{\mathrm{WT}}$ ) with or without proviral DNA pNL4-3. FLAG complexes were then immunoprecipitated and the eluted complexes were assessed for FLAG (UPF1), UPF2, UPF3b, Gag and GAPDH by Western blotting analysis (Figure 6B). We observed that UPF3b and UPF2 co-immunoprecipitated with UPF $1^{\text {WT }}$ but UPF3b co-immunoprecipitated with UPF1 to a several-fold greater extent in HIV-1 conditions in which little change in the association between UPF1 and UPF2 was observed. These observations indicate that the interaction between UPF3b and UPF1 is promoted and favoured by HIV-1, in contrast to that found to occur between UPF1 and UPF2, which is not modulated by HIV-1 and could explain the exclusion of UPF2 from the HIV-1 RNP [5].

A

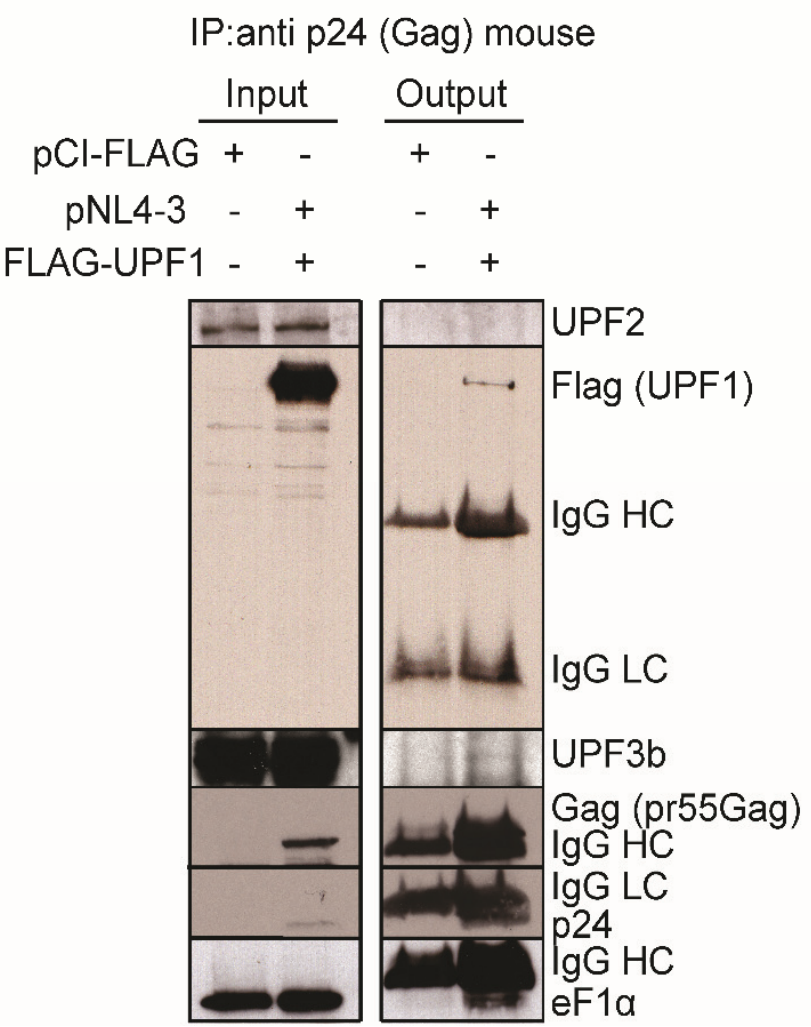

B

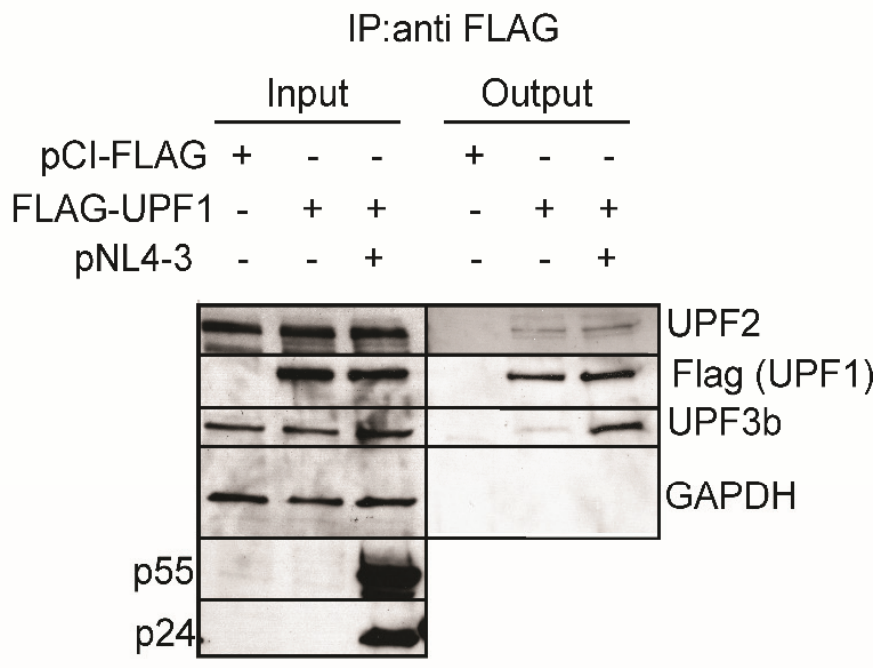

Figure 6. UPF 2 is absent from the HIV-1 Gag RNP and UPF3b co-immunoprecipitates with UPF1 under HIV-1 conditions. (A) HeLa cells were mock transfected with pCI-FLAG or cotransfected with proviral pNL4-3 DNA and FLAG-UPF1 ${ }^{\text {WT }}$. At $30 \mathrm{~h}$ post-transfection, cells were harvested and Gag was immunoprecipitated using a monoclonal anti-p24 antiserum as described in Materials and Methods. Western blot analysis for FLAG-UPF1, UPF3b, Gag (pr55 ${ }^{\mathrm{Gag}}$ ), UPF2, and eF1 $\alpha$ (positive control) was performed. HC: IgG Heavy Chain. LC: IgG Light Chain. Results are representative of two experiments. (B) HeLa cells were mock transfected with pCI-FLAG or FLAG-UPF1 or co-transfected with HIV-1 pNL4-3 DNA. Cells were harvested and FLAG-UPF1 was immunoprecipitated. Input lysates and bound complexes were analyzed by western blotting for UPF2, FLAG-UPF1, UPF3b, GAPDH (loading and output control) and Gag (pr55 ${ }^{\mathrm{Gag}}$ ). Results shown are representative of three independent experiments. HC: IgG Heavy Chain, LC: IgG Light Chain. 


\subsection{UPF2, in Complex with UPF1, Blocks vRNA Nucleocytoplasmic Export}

The major binding partners of UPF2 are UPF1, UPF3aL and UPF3b. Our previous results demonstrate that UPF3b overexpression did not elicit any changes to vRNA or Gag levels, and UPF2-binding mutants of UPF1 still upregulated Gag levels [5]. In addition, HIV-1 vRNA localized in a punctate pattern in the cytoplasm of cells (Figure 7A) consistent with our earlier work [5] and UPF3b expression had no marked effect on this localization pattern (Figure 7B). Since it was shown that UPF3aL and UPF3b undergo a unidirectional regulation that is dependent on their binding to UPF2 [49] and we had previously shown that UPF3b had no effect on vRNA metabolism [5], we wondered whether overexpressed UPF3aL could have a role similar to its paralog, UPF3b. To our surprise, UPF3aL expression blocked vRNA nucleocytoplasmic export resulting in a phenotype similar to that found when Rev is absent (Rev-, Figure 7C; compare with Figure 1C), in contrast, to the localization phenotype obtained with UPF3b (Figure 7B). Since we have shown that UPF1 is involved in vRNA export, we determined how these UPF proteins bound UPF1. HeLa cells were transfected with FLAG DNA (empty vector pCI-FLAG) with or without proviral pNL4.3 and with UPF3aL (FLAG-UPF3aL) or UPF3b (FLAG-UPF3b) with proviral pNL4-3. FLAG complexes were then immunoprecipitated with FLAG-conjugated agarose beads and the eluted complexes were assessed for FLAG, UPF1 and UPF2 by western blotting analysis. UPF2 and UPF1 co-immunoprecipitated with both UPF3b and UPF3aL (Figure 7H), without any change in vRNA levels [73].

UPF3b and UPF3aL undergo a unidirectional regulation which is dependent on the availability of UPF2. Since UPF2 is excluded from the HIV-1 RNP, we hypothesized that UPF2 binding to UPF3aL with UPF1 mediates the blockade to vRNA nuclear export. We therefore overexpressed the UPF3aL mutant, FLAG-UPF3aS, that does not interact with UPF2 but is a component of a complex containing UPF1 (but not UPF2), and examined vRNA localization by FISH/IF co-analyses (Figure 7D). In contrast with what was observed with UPF3aL, overexpression of FLAG-UPF3aS did not lead to the nuclear localization of the vRNA (Figure 7D). We then overexpressed FLAG-UPF3aL and FLAG-UPF3aS with proviral pNL4-3 DNA, immunoprecipitated these complexes using an anti-FLAG antibody and blotted for the presence of FLAG, UPF1 and UPF2 (Figure 7I). As expected, UPF1 co-immunoprecipitated with both UPF3aL and UPF3aS, but the UPF2 binding mutant, UPF3aS, did not pull down UPF2. Thus, it seems that UPF2, UPF3aL and UPF1 in complex are detrimental to HIV-1 expression by trapping the vRNA in the nucleus, while UPF3aS in complex with UPF1 does not have this effect due to the fact that it does not bind UPF2. Given that our results point to a negative regulation of vRNA export by UPF2, we decided to express wild type UPF2 (FLAG-UPF2) and a mutant of UPF2 which does not bind UPF1 (FLAG-UPF2 ${ }^{1-1096}$, which has a deletion at the C-terminus) with proviral DNA pNL4-3 and assessed vRNA localization by FISH/IF co-analyses (Figure 7E,F). Wild type UPF2 blocked vRNA nuclear export (Figure 7E), similar to what was found for UPF3aL. The C-terminal mutant UPF2 ${ }^{1-1096}$ exhibiting a complete loss of UPF1 binding failed to block vRNA export (Figure 7F). A point mutant of UPF2 (UPF2 ${ }^{1173}$ ), which exhibits an incomplete association to UPF1, resulted in a clear 50\% phenotype between nuclear and cytoplasmic localization of the vRNA ([73], see below). These results show that UPF2 blocks vRNA nucleocytoplasmic export by associating with UPF1.

Since the C-terminal domain of UPF2 binds the N-terminal CH-domain of UPF1 [48], we therefore decided to confirm that the deletion mutant UPF2 ${ }^{1-1096}$ does not bind UPF1. Our results show that all of 
the UPF2 constructs immunoprecipitated with UPF3b but bound UPF1 differentially (Figure 7J). UPF1 immunoprecipitated with wild type UPF2 but weakly with, UPF2 ${ }^{1173}$ explaining in part why there was only a partial 50\% blockade to nuclear export of the vRNA ( $n>50$, in 3 independent experiments; [73]), despite a quantitative immunoprecipitation of FLAG-UPF2 proteins. On the other hand, UPF1 failed to immunoprecipitate with the C-terminal mutant of UPF2 (UPF2 ${ }^{1-1096}$; Figure 7J) correlating with the loss in the ability to block the nuclear export of the vRNA. There was no significant change in vRNA levels under these conditions (Figure 7J, bottom). Importantly, it was shown that UPF2 ${ }^{1-1096}$ was still able to bind UPF3aL and UPF3b despite its inability to bind UPF1 [48]. The characteristics and binding partners of UPF3 and UPF2 wild type and mutants used as well as a summary of their effects on Rev+ HIV-1 vRNA export are summarized in Table 2. These results demonstrate that the UPF3aL/UPF2-UPF1 association blocks vRNA nuclear export (Figure 7G) and that HIV-1 excludes UPF2 from its binding to UPF1 in order for UPF1 to promote HIV-1 vRNA export together with Rev, CRM1 and DDX3.

A

B

D

m

之

E
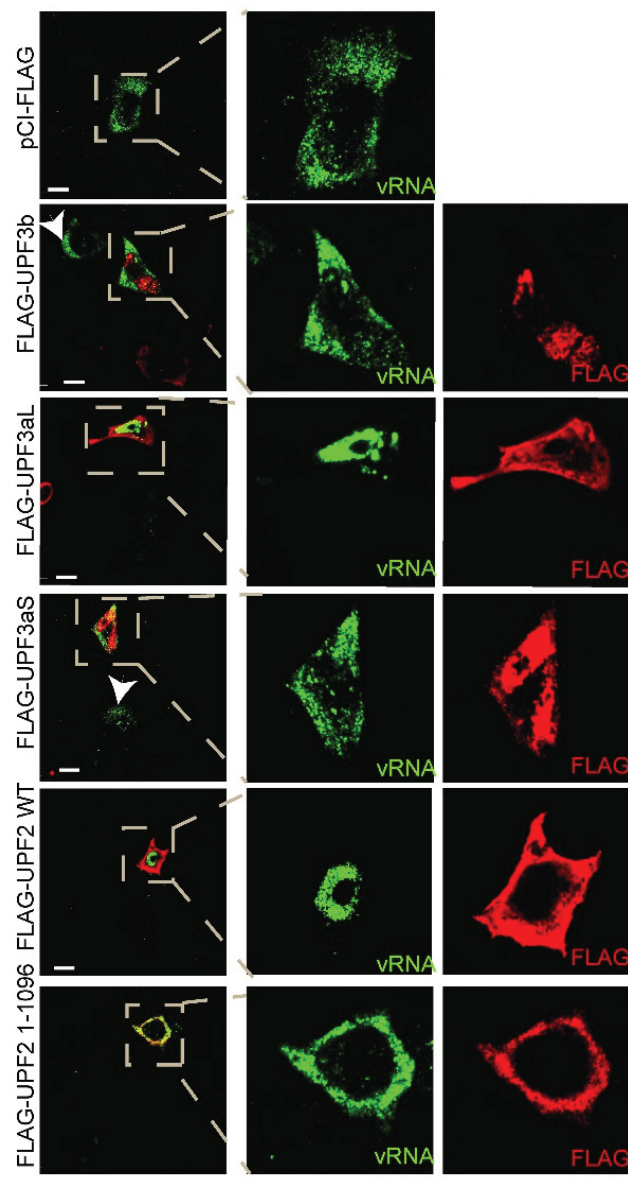

IP:anti FLAG

$\mathrm{H}$

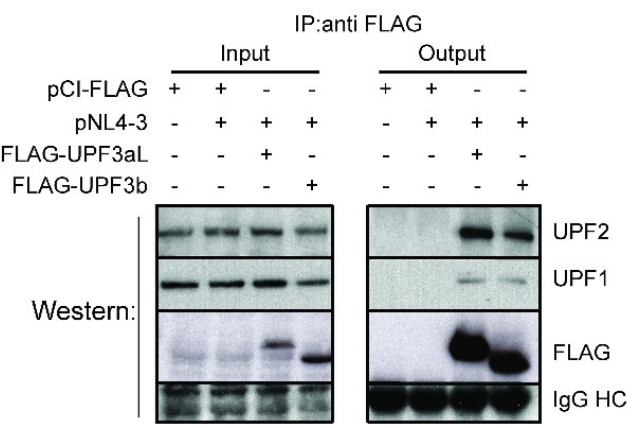

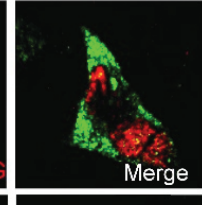

G

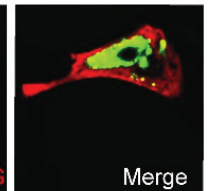

Merge
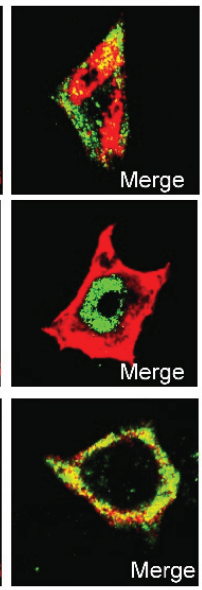

I

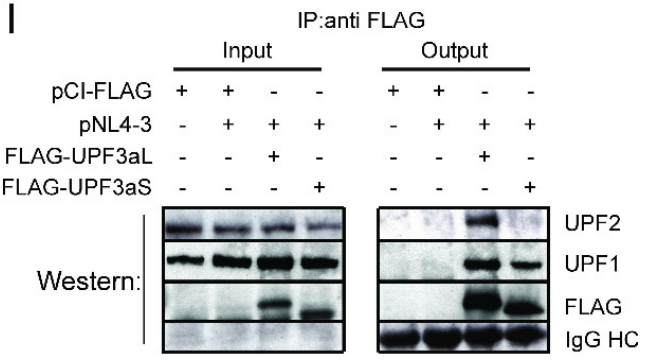

Figure 7. Cont. 


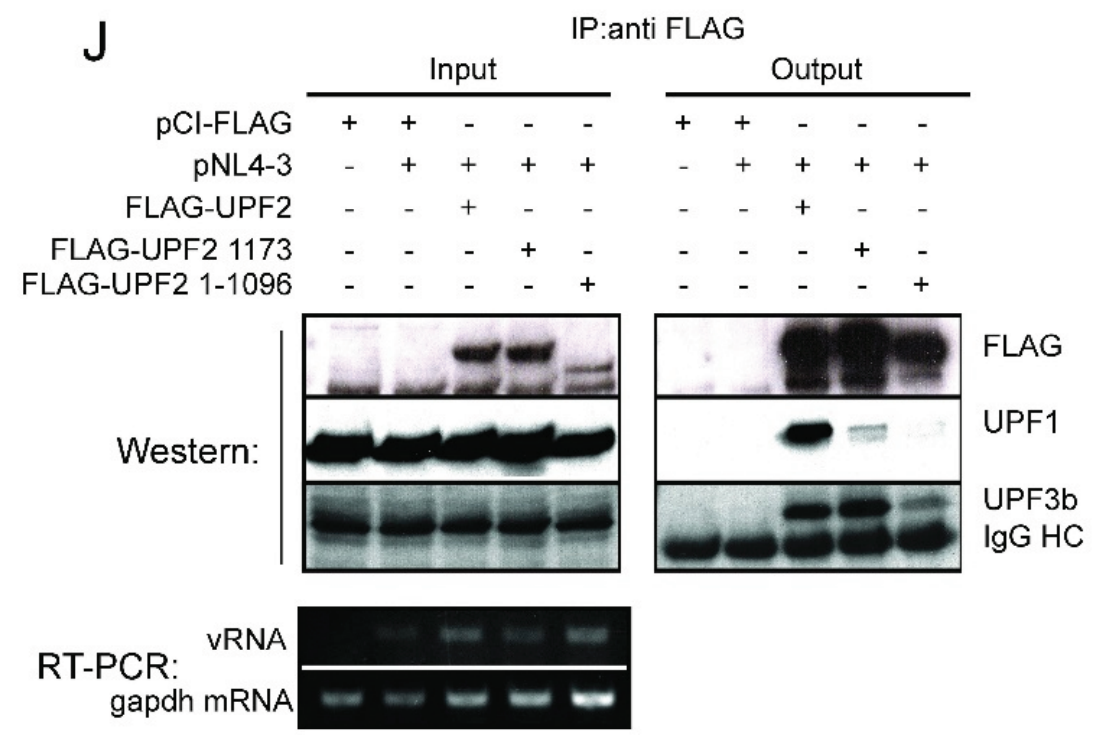

Figure 7. UPF3aL and UPF2 block vRNA export by associating to UPF1 as determined by FISH/IF and co-immunoprecipitation analyses. HeLa cells were co-transfected with pNL4-3 with pCI-FLAG (A); and FLAG-tagged UPF proteins: UPF3b (B); UPF3aL (C); UPF3aS (D); UPF2 (E); or UPF2 1-1096 (F); and processed for FISH/IF co-analyses to determine the localization of vRNA (green) and UPF proteins (B-F) (red); White arrowheads identify cells transfected with pNL4-3 alone. (G) Dunnett post-test analyses on vRNA localization results \pm SEM values compared to the controls pNL4-3+pCI-FLAG: $* * * p<0.001$. Co-immunoprecipitation analyses confirm that UPF2 association blocks vRNA export. HeLa cells were mock transfected with pCI-FLAG or co-transfected with HIV-1 pNL4-3 proviral DNA and pCI-FLAG or FLAG-UPF3aL or FLAG-UPF3b (H). HeLa cells were transfected as in (H) but FLAG-UPF3aS was included instead of FLAG-UPF3b (I). HeLa cells were transfected as in (H) except that FLAG-tagged UPF2 proteins were co-transfected: UPF2WT or UPF21173 or UPF21-1096 (J). At 48 h post-transfection, cells were harvested and FLAG-tagged UPF proteins were individually immunoprecipitated as described in Materials and Methods. Input and bound (Output) complexes were analyzed by western blotting for UPF proteins as indicated. The abundance of vRNA and gapdh RNA was estimated by RT-PCR (in Input only). Results shown are representative of 2 independent experiments.

Table 2. UPF2 and UPF3 plasmids.

\begin{tabular}{|c|c|c|c|c|c|c|}
\hline \multirow{2}{*}{$\begin{array}{l}\text { UPF } \\
\text { Plasmids } \\
\text { Used }\end{array}$} & \multirow[b]{2}{*}{ Deleted Region } & \multirow[b]{2}{*}{ Protein Localization } & \multirow[b]{2}{*}{ Characteristics } & \multirow{2}{*}{$\begin{array}{l}\text { Effect on HIV-1 RNA } \\
\text { Export (Rev+ } \\
\text { Conditions) }\end{array}$} & \multicolumn{2}{|c|}{ Interactions } \\
\hline & & & & & UPF1 & UPF2 \\
\hline UPF3b & NA & $\begin{array}{l}\text { Nuclear and } \\
\text { cytoplasmic }\end{array}$ & $\begin{array}{l}\text { Forms complexes with UPF1, } \\
2 \text { and } 3 \text { aL }[31,47,48] \text {; regulates } \\
\text { UPF3A levels [49] }\end{array}$ & $\begin{array}{l}\text { No effect on Rev+ } \\
\text { export [5] and this study }\end{array}$ & Yes & Yes \\
\hline UPF3aL & NA & $\begin{array}{l}\text { Nuclear and } \\
\text { cytoplasmic }\end{array}$ & $\begin{array}{l}\text { Forms complex with } \\
\text { phospho-UPF1-UPF2 }[31,47] \\
\text { levels regulated by UPF3b, } \\
\text { UPF2 [49] }\end{array}$ & $\begin{array}{l}\text { Negatively affects Rev }+ \\
\text { export (this study) }\end{array}$ & Yes & Yes \\
\hline
\end{tabular}


Table 2. Cont.

\begin{tabular}{|c|c|c|c|c|c|c|}
\hline \multirow{2}{*}{$\begin{array}{l}\text { UPF } \\
\text { Plasmids } \\
\text { Used }\end{array}$} & \multirow{2}{*}{ Deleted Region } & \multirow{2}{*}{$\begin{array}{l}\text { Protein } \\
\text { Localization }\end{array}$} & \multirow{2}{*}{ Characteristics } & \multirow{2}{*}{$\begin{array}{l}\text { Effect on HIV-1 RNA } \\
\text { Export (Rev+ } \\
\text { Conditions) }\end{array}$} & \multicolumn{2}{|c|}{ Interactions } \\
\hline & & & & & UPF1 & UPF2 \\
\hline UPF3aS & $\begin{array}{l}\text { Exon4 from } \\
\text { UPF3aL residues } \\
\text { 117-149 }\end{array}$ & $\begin{array}{l}\text { Nuclear and } \\
\text { cytoplasmic }\end{array}$ & $\begin{array}{l}\text { Exon } 4 \text { from UPF3aL [31]; forms } \\
\text { complex with PUPF1-SMG5/7 [31]; } \\
\text { does not bind UPF2 }[31,48]\end{array}$ & $\begin{array}{l}\text { No effect on Rev+ export } \\
\text { (this study) }\end{array}$ & Yes & No \\
\hline UPF2 ${ }^{\text {WT }}$ & NA & Cytoplasmic* & $\begin{array}{l}\text { Forms complex with UPF1, } 3 \text { aL, } \\
\text { 3b }[31,47-49]\end{array}$ & $\begin{array}{l}\text { Negatively affects Rev }+ \\
\text { export (this study) }\end{array}$ & Yes & NA \\
\hline UPF $2^{1-1096}$ & $\begin{array}{l}\text { C-terminal } \\
\text { deletion } 1096 \\
\text { to } 1272\end{array}$ & Cytoplasmic & $\begin{array}{l}\text { C-terminal deletion }[48,53] \text {; does not } \\
\text { bind UPF1 }[48,53]\end{array}$ & $\begin{array}{l}\text { No effect on } \mathrm{Rev}+\text { export } \\
\text { (this study) }\end{array}$ & No & NA \\
\hline
\end{tabular}

NA: Not applicable; * Associates to nuclear proteins and may be resident in nucleus.

\section{Discussion}

In this study, we identify a novel function for UPF1 in regulating the HIV-1 vRNA fate. UPF1 appears to be acting at multiple levels of control during vRNA biogenesis. First, our results indicate that UPF1 homes the RRE-containing vRNA (Figures 1 and 2). Second, considering that UPF1 overcomes nuclear HIV-1 vRNA retention in the absence of Rev (Figure 1), UPF1 thus represents a dominant adaptor protein that can associate to the vRNA in both $\mathrm{Rev}^{-}$and $\mathrm{Rev}+$ conditions to promote nuclear export. This appears to be in the context of a nuclear HIV-1 export UPF1-containing RNP (Figure 4). Indeed, HIV-1 may have evolved the means to efficiently program the trafficking of its mRNAs by co-opting UPF1 function. Nevertheless, UPF1 only affects RRE-mediated, but not CTE-mediated export, similar to what was found for DDX3 [12]. The other similarity includes the observation that UPF1, as well as DDX3, bind de novo synthesized vRNA in the nucleus [44] and recent results show that UPF1 selectively associates to long 3'-UTR substrates [39], of which the vRNA is one. Interestingly, it was shown that UPF1 associates with transcription sites [84]. Not only does Rev cause the relocalization of CRM1 to transcription sites but Rev-CRM1-RRE-containing RNAs are released as one complex from these transcription sites [4]. Thus, it is likely that UPF1 is part of this complex since it was shown to be present at the transcription sites, associated with de novo synthesized HIV-1 vRNA and is part of a complex with Rev, CRM1, DDX3 and the HIV-1 vRNA.

Our results not only reveal that UPF1 is found in two different HIV-1 RNP complexes, one in which DDX3, CRM1, Nup62 and Rev are found, and another in which Gag is present (Figure 4), but also provide further support for both nuclear and cytoplasmic functions of UPF1 in HIV-1 replication. In a recent proteomic screen, UPF1 and UPF3b were found in association with Gag or Rev, amongst other viral proteins, strengthening the validity of our results [85] even though we have not observed UPF3b association with HIV-1 vRNA [73]. In the nucleus, UPF1 most likely enhances the association of the HIV-1 vRNA with export factors and then accompanies the vRNA into the cytoplasm where it enhances stability and translation independently of its nuclear remodelling function [5]. In addition, UPF1 has been found in virus particles $[61,86]$ and purified viral cores [76] whose presence can be mediated by its interaction with the vRNA. Finally, our ongoing work using latently infected model cell systems reveals 
that when latent provirus is reactivated, a several-fold increase in UPF1 expression levels is the result, concomitant with the burst in Gag expression, while the levels of UPF2 and UPF3aL did not change significantly [73].

Our data suggest that nucleo-cytoplasmic shuttling of UPF1 requires the NES sequence present in the helicase, which would be expected to bind the NES-binding cleft in CRM1 competing with Rev for binding. However, recent studies by Frankel and colleagues showed that CRM1 forms a dimer within the Rev/RRE nuclear export complex [87] raising the possibility for the formation of a Rev/CRM1-CRM1/UPF1 complex. Moreover, based on our structural modeling and previous data, it is tempting to speculate that this viral nuclear export complex would be stabilized by Rev-UPF1, DDX3-UPF1 and DDX3-CRM1 [12] interactions. Indeed, a role for UPF1 in RNA export is not without precedence. Yeast-two hybrid studies have shown that UPF1 interacts with nucleoporins Nup100/116 (the mammalian homolog is Nup98), suggesting that this interaction ensures the association of UPF1 with the newly synthesized mRNAs $[44,75,88]$. Interestingly, Nup98 is also a cofactor of CRM1/Rev as well as an important nucleoporin involved in vRNA export [89]. Further studies revealed that Nup62 is a component of the Rev RNP [10] and recent work demonstrates that Nup62 is a host component involved in mediating vRNA nucleocytoplasmic export [15]. Ataxia-telangiectasia-mutated kinase (ATM), while involved in HIV-1 DNA integration and DNA damage, also promotes vRNA nucleocytoplasmic export by activating Rev [90] and ATM phosphorylates UPF1 [91]. Finally, recent work demonstrates co-transcriptional assembly of Rev, CRM1, and RRE-containing RNAs at the site of transcription, and now likely with the participation of UPF1, in one single export complex [4]. Future work will be needed to determine if the post-translational modification of UPF1 is required for its activity in nucleocytoplasmic export [90,92].

The second major finding is that UPF2-UPF3aL acts as a negative regulator of vRNA nuclear export. UPF2 was first found to be excluded from the HIV-1 RNP and mutants of UPF1 that do not bind UPF2 still upregulated vRNA translation [5]. We extended these findings to show that, whereas UPF2 expression blocks vRNA nucleocytoplasmic export, UPF2 mutants that do not bind UPF1 lose this ability (Figure 7). Moreover, our in silico analyses allowed us to gain insights on the molecular mechanism of the UPF2 exclusion, which is possibly mediated by the binding of Rev to the CH-domain of UPF1 at a site that overlaps with the UPF2 binding site (Figure 5). The regulatory circuit we described here also implicates UPF3a. Earlier work demonstrated that both isoforms of UPF3a (UPF3aL and UPF3aS) are in different complexes with UPF1 [31] termed the "post-phosphorylation complex" (UPF3aL-UPF2 with P-UPF1) and the "pre-dephosphorylation complex" (UPF3aS-SMG5/7-PP2A-P-UPF1) [31]. The presence of both of these complexes in the cell can explain why both UPF3a isoforms, which differ in their ability to bind UPF2, have differential effects on vRNA export. UPF3aL overexpression leads to the recruitment of both UPF2 and UPF1, blocking vRNA export, while UPF3aS only recruits UPF1 but excludes UPF2, resulting in little effect on nucleocytoplasmic vRNA export (Figure 7). The strict exclusion of UPF2 imposed by HIV-1 shown here and earlier [5] would also enhance UPF1's ability to bind RNA, perhaps the vRNA in infected cells, by a conformational switch induced by this association [28] resulting in enhanced stabilization, nucleocytoplasmic export and translatability of the vRNA.

Although the precise function of UPF2 in the fate of HIV-1 vRNA remains incompletely understood, its expression levels may directly influence the availability of UPF1. While UPF1 has been shown to shuttle between the nucleus and cytoplasm, UPF2 has been reported to be strictly cytoplasmic [21]. It is therefore conceivable that UPF2 prevents UPF1 from being imported to the nucleus, where it could enhance viral 
RNA production and export (see model presented in Figure 8). Nevertheless, several independent studies show that UPF2 localizes to nuclear fractions [31,93,94] and that UPF2 associates with UPF3aL (a nucleocytoplasmic shuttling protein), hinting at the possibility that UPF2 might also be a component of nuclear RNPs. Moreover, NMD and SMD are believed to be competitive mechanisms because UPF2 and Staufen1 bind to the same region in UPF1 (Figure 1A and [95]). Since UPF1 is a component of the Staufen1/HIV-1 RNP complex that excludes UPF2 [45], it is possible that HIV-1 mediates the association between UPF1 and Staufen1, occluding UPF2's ability to associate to UPF1 [95]. Hence, we propose that high expression levels of UPF2 would lead to the formation of UPF1-containing cytoplasmic complexes and limit the availability of UPF1 to the nucleus, resulting in a block to HIV-1 vRNA export that depends on UPF1 nucleocytoplasmic shuttling.

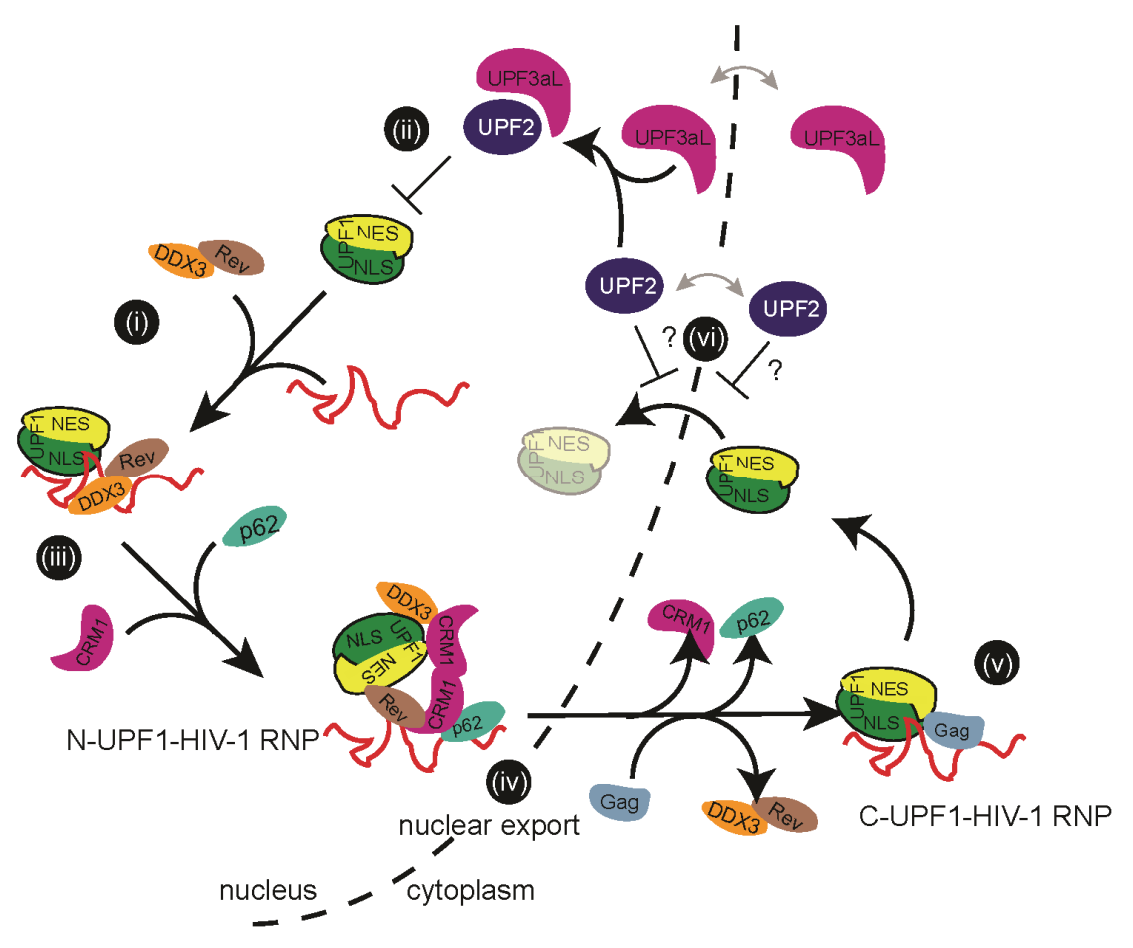

Figure 8. Proposed model for UPF1-mediated vRNA nucleocytoplasmic export. During the HIV-1 expression phase, the shuttling ability of UPF1 is important for vRNA nucleocytoplasmic export. In the nucleus, UPF1 can be recruited to the vRNA via its C-terminal domain (including the NLS domain, Figure 1A) and acts as an adaptor protein to bridge the nuclear HIV-1 RNP with export factors through, most likely, its NES region (i). However, UPF3aL can potentially block the recruitment of UPF1 to prevent the assembly of an export-competent nuclear RNP by recruiting UPF2 (ii). Once assembled, the UPF1 nuclear export complex, composed of the vRNA, UPF1, Rev, CRM1, DDX3 and DDX1 then associates with nucleoporins Nup98 and/or Nup214, as well as Nup62 to form a nuclear complex ( $N$-UPF1-HIV-1 RNP, (iii)). This complex can then be efficiently exported (iv). Once in the cytoplasm, several factors including UPF1 recycle back to their original locations. UPF1 can either act in the context of a cytoplasmic RNP (C-UPF1-HIV-1 RNP) to promote vRNA stability and utilization (translation) to ensure Gag synthesis (v), or, according to the current results in this manuscript, escapes interaction with UPF2 (vi) within the cytoplasm or nucleus (“?” indicates open question) to control vRNA nucleocytoplasmic export. 
The findings presented in this manuscript do not represent the first UPF2-independent function of UPF1 [25,41]. Our current model proposes that immediately following viral transcription, UPF1 is recruited to the vRNA via association to the NLS domain, the helicase IV-VI and/or C-terminal domains and to steer this intron-containing mRNA away from the nuclear RNA quality control and surveillance machinery (Figure 8, step (i)). UPF1 can then serve as an adaptor protein for Rev/CRM1-mediated vRNA export. The immediate recruitment of UPF1 is in-line with earlier [44] as well as recent findings on UPF1's ability to bind long, 3'-UTRs [46] that precedes NMD [39] and on the early recruitment of Rev and CRM1 to sites of HIV-1 transcription [4]. The nuclear RNP (N-UPF1-HIV-1 RNP) will then acquire additional nuclear trafficking proteins including DDX3, DDX1, eIF5A, amongst others, that are important for vRNA nuclear export, and will position itself for nuclear export through the nuclear pore (Figure 8, steps (iii-iv)). The RNP engages several nuclear pore proteins, such as Nup98, Nup214 and/or Nup62, resulting in the efficient export and cytoplasmic localization of the vRNA. At this point neither the precise makeup of the viral nuclear export RNP is completely characterized nor do we have a full understanding of the direct and indirect intermolecular interactions that occur.

UPF2 does not play a functional role in several UPF1-containing mRNPs such as the UPF2-independent NMD RNP [41], the UPF1 RNP that sits on structured 3'-UTRs [96] and another that regulates histone mRNA stability during cell replication [25]. Our results suggest that UPF2 is excluded from HIV-1 RNPs through the selective or antagonistic interactions with viral and cellular proteins, such as Rev and/or Staufen 1 that will invariably impact function. UPF2's association to UPF1, for example, impacts UPF1 structure and influences enzymatic and other functions, so this scenario seems to be the most likely [28,53,97]. This might also be true for UPF1's newly characterized role in HIV-1 reverse transcription of the vRNA early in HIV-1 infection, at which stage UPF2 still has no role [86]. Anti-viral strategies using the Revtransdominant RevM10 to block vRNA nuclear export have failed because of the development of resistance, so targeting of Rev alone will likely not be sufficient. Therefore, cofactors such as DDX3 and UPF1 that assemble with and aid in Rev function remain viable targets [18]. Since UPF1 is required for vRNA stability and translation with an added functionality in the control of nucleocytoplasmic RNA export that implicates other UPF proteins, the targeting of this regulatory circuit could represent a suitable and multi-pronged approach that will contribute to the arsenal that we use to treat and eventually cure HIV-1 infection.

\section{Conclusions}

HIV-1 commandeers UPF host proteins for critical steps of the viral replication cycle. In this manuscript we demonstrate that HIV-1 ensures nuclear export of the genomic RNA by the recruitment of UPF1 but by the exclusion of UPF2.

\section{Acknowledgements}

We thank Jens Lykke-Andersen, Harry Dietz, William Rigby, Alan Cochrane, Ruth Brack-Werner, Bryan Cullen and Paul Beniasz for generous provision of antisera and clones and Reza Sadaie, Bruce Chesebro and the NIH AIDS Reference and Reagent Program for reagents. 


\section{Author Contributions}

Lara Ajamian and Andrew J. Mouland conceived of the study and designed experiments with participation of Niels H. Gehring and Andreas E. Kulozik; Lara Ajamian carried out most of the experiments; Kishanda Vyboh, Karen Abel and Shringar Rao performed IP and some imaging experiments; Francisco García-de-Gracia and Ricardo Soto-Rifo performed virtual modeling; Lara Ajamian and Andrew J. Mouland drafted the manuscript. All authors read and approved the final manuscript.

Lara Ajamian was a recipient of a Canadian Institutes of Health Research (CIHR) Doctoral Research Award; Francisco García-de-Gracia was a recipient of a Conicyt Doctoral fellowship; Andrew J. Mouland was supported by a McGill University Fraser, Monat and McPherson Scholarship. This work was supported by grants from the German Research Foundation (DFG) and the Manfred Lautenschläger Stiftung to Andreas E. Kulozik, the German Research Foundation (DFG grant GE2014/2-1 and SFB635, project B6) to Niels H. Gehring, Conicyt-Chile through the Fondecyt Initiation into Research Program (No 11121339) to Ricardo Soto-Rifo and grants from the CIHR (MOP-38111 and MOP-56974) and The Canadian HIV Cure Enterprise Team Grant HIG-133050 to Andrew J. Mouland, the latter funded in partnership with CANFAR and IAS.

\section{Conflicts of Interest}

The authors declare that they have no competing interests.

\section{References}

1. Cullen, B.R. Nuclear mRNA export: Insights from virology. Trends Biochem. Sci. 2003, 28, 419-424.

2. Grunwald, D.; Singer, R.H.; Rout, M. Nuclear export dynamics of RNA-protein complexes. Nature 2011, 475, 333-341.

3. Braun, I.C.; Herold, A.; Rode, M.; Conti, E.; Izaurralde, E. Overexpression of tap/p15 heterodimers bypasses nuclear retention and stimulates nuclear mRNA export. J. Biol. Chem. 2001, 276, 20536-20543.

4. Nawroth, I.; Mueller, F.; Basyuk, E.; Beerens, N.; Rahbek, U.L.; Darzacq, X.; Bertrand, E.; Kjems, J.; Schmidt, U. Stable assembly of HIV-1 export complexes occurs cotranscriptionally. RNA 2014, 20, 1-8.

5. Ajamian, L.; Abrahamyan, L.; Milev, M.; Ivanov, P.V.; Kulozik, A.E.; Gehring, N.H.; Mouland, A.J. Unexpected roles for UPF1 in HIV-1 RNA metabolism and translation. RNA 2008, 14, 914-927.

6. Mocquet, V.; Neusiedler, J.; Rende, F.; Cluet, D.; Robin, J.P.; Terme, J.M.; Duc Dodon, M.; Wittmann, J.; Morris, C.; le Hir, H.; et al. The human T-lymphotropic virus type 1 tax protein inhibits nonsense-mediated mRNA decay by interacting with INT6/EIF3E and UPF1. J. Virol. 2012, $86,7530-7543$.

7. Nakano, K.; Ando, T.; Yamagishi, M.; Yokoyama, K.; Ishida, T.; Ohsugi, T.; Tanaka, Y.; Brighty, D.W.; Watanabe, T. Viral interference with host mRNA surveillance, the nonsense-mediated mRNA decay (NMD) pathway, through a new function of HTLV-1 Rex: Implications for retroviral replication. Microbes Infect. 2013, 15, 491-505. 
8. Purcell, D.F.; Martin, M.A. Alternative splicing of human immunodeficiency virus type $1 \mathrm{mRNA}$ modulates viral protein expression, replication, and infectivity. J. Virol. 1993, 67, 6365-6378.

9. Malim, M.H.; Hauber, J.; Le, S.Y.; Maizel, J.V.; Cullen, B.R. The HIV-1 Rev trans-activator acts through a structured target sequence to activate nuclear export of unspliced viral mRNA. Nature 1989, 338, 254-257.

10. Hofmann, W.; Reichart, B.; Ewald, A.; Muller, E.; Schmitt, I.; Stauber, R.H.; Lottspeich, F.; Jockusch, B.M.; Scheer, U.; Hauber, J.; et al. Cofactor requirements for nuclear export of Rev response element (RRE) - and constitutive transport element (CTE)-containing retroviral RNAs: An unexpected role for actin. J. Cell Biol 2001, 152, 895-910.

11. Kjems, J.; Askjaer, P. Rev protein and its cellular partners. Adv. Pharmacol. 2000, 48, 251-298.

12. Yedavalli, V.S.; Neuveut, C.; Chi, Y.H.; Kleiman, L.; Jeang, K.T. Requirement of DDX3 dead box RNA helicase for HIV-1 Rev-RRE export function. Cell 2004, 119, 381-392.

13. Modem, S.; Badri, K.R.; Holland, T.C.; Reddy, T.R. Sam68 is absolutely required for Rev function and HIV-1 production. Nucleic Acids Res. 2005, 33, 873-879.

14. Reddy, T.R.; Tang, H.; Xu, W.; Wong-Staal, F. Sam68, RNA helicase a and tap cooperate in the post-transcriptional regulation of human immunodeficiency virus and type D retroviral mRNA. Oncogene 2000, 19, 3570-3575.

15. Monette, A.; Pante, N.; Mouland, A.J. HIV-1 remodels the nuclear pore complex. J. Cell Biol. 2011, 193, 619-631.

16. Modem, S.; Reddy, T.R. An anti-apoptotic protein, HAX-1, inhibits the HIV-1 Rev function by altering its sub-cellular localization. J. Cell Physiol. 2008, 214, 14-19.

17. Zhou, Y.; Rong, L.; Zhang, J.; Aloysius, C.; Pan, Q.; Liang, C. Insulin-like growth factor II mRNA binding protein 1 modulates Rev-dependent human immunodeficiency virus type 1 RNA expression. Virology 2009, 393, 210-220.

18. Radi, M.; Falchi, F.; Garbelli, A.; Samuele, A.; Bernardo, V.; Paolucci, S.; Baldanti, F.; Schenone, S.; Manetti, F.; Maga, G.; et al. Discovery of the first small molecule inhibitor of human DDX3 specifically designed to target the RNA binding site: Towards the next generation HIV-1 inhibitors. Bioorg. Med. Chem. Lett. 2012, 22, 2094-2098.

19. Nicholson, P.; Yepiskoposyan, H.; Metze, S.; Orozco, R.Z.; Kleinschmidt, N.; Muhlemann, O. Nonsense-mediated mRNA decay in human cells: Mechanistic insights, functions beyond quality control and the double-life of nmd factors. Cell Mol. Life Sci. 2010, 67, 677-700.

20. Brogna, S.; Wen, J. Nonsense-mediated mRNA decay (NMD) mechanisms. Nat. Struct. Mol. Biol. 2009, 16, 107-113.

21. Mendell, J.T.; ap Rhys, C.M.; Dietz, H.C. Separable roles for RENT1/HUPF1 in altered splicing and decay of nonsense transcripts. Science 2002, 298, 419-422.

22. Isken, O.; Maquat, L.E. The multiple lives of nmd factors: Balancing roles in gene and genome regulation. Nat. Rev. Genet. 2008, 9, 699-712.

23. Ciaudo, C.; Bourdet, A.; Cohen-Tannoudji, M.; Dietz, H.C.; Rougeulle, C.; Avner, P. Nuclear mRNA degradation pathway(s) are implicated in xist regulation and $\mathrm{X}$ chromosome inactivation. PLoS Genet. 2006, 2, e94.

24. Kim, Y.K.; Furic, L.; Desgroseillers, L.; Maquat, L.E. Mammalian staufen1 recruits UPF1 to specific mRNA 3'-UTRs so as to elicit mRNA decay. Cell 2005, 120, 195-208. 
25. Azzalin, C.M.; Lingner, J. The human RNA surveillance factor UPF1 is required for s phase progression and genome stability. Curr. Biol. 2006, 16, 433-439.

26. Chawla, R.; Redon, S.; Raftopoulou, C.; Wischnewski, H.; Gagos, S.; Azzalin, C.M. Human UPF1 interacts with TPP1 and telomerase and sustains telomere leading-strand replication. EMBO J. 2011, 30, 4047-4058.

27. Agranat, L.; Raitskin, O.; Sperling, J.; Sperling, R. The editing enzyme ADAR1 and the mRNA surveillance protein HUPF1 interact in the cell nucleus. Proc. Natl. Acad. Sci. USA 2008, 105, 5028-5033.

28. Chamieh, H.; Ballut, L.; Bonneau, F.; le Hir, H. NMD factors UPF2 and UPF3 bridge UPF1 to the exon junction complex and stimulate its RNA helicase activity. Nat. Struct. Mol. Biol. 2008, 15, 85-93.

29. Ivanov, P.V.; Gehring, N.H.; Kunz, J.B.; Hentze, M.W.; Kulozik, A.E. Interactions between UPF1, ERFS, PABP and the exon junction complex suggest an integrated model for mammalian NMD pathways. EMBO J. 2008, 27, 736-747.

30. Kashima, I.; Yamashita, A.; Izumi, N.; Kataoka, N.; Morishita, R.; Hoshino, S.; Ohno, M.; Dreyfuss, G.; Ohno, S. Binding of a novel smg-1-UPF1-ERF1-ERF3 complex (SURF) to the exon junction complex triggers UPF1 phosphorylation and nonsense-mediated mRNA decay. Genes Dev. 2006, 20, 355-367.

31. Ohnishi, T.; Yamashita, A.; Kashima, I.; Schell, T.; Anders, K.R.; Grimson, A.; Hachiya, T.; Hentze, M.W.; Anderson, P.; Ohno, S. Phosphorylation of HUPF1 induces formation of mRNA surveillance complexes containing HSMG-5 and HSMG-7. Mol. Cell 2003, 12, 1187-1200.

32. Bhattacharya, A.; Czaplinski, K.; Trifillis, P.; He, F.; Jacobson, A.; Peltz, S.W. Characterization of the biochemical properties of the human UPF1 gene product that is involved in nonsense-mediated mRNA decay. RNA 2000, 6, 1226-1235.

33. Rebbapragada, I.; Lykke-Andersen, J. Execution of nonsense-mediated mRNA decay: What defines a substrate? Curr. Opin. Cell Biol. 2009, 21, 394-402.

34. Bruno, I.G.; Karam, R.; Huang, L.; Bhardwaj, A.; Lou, C.H.; Shum, E.Y.; Song, H.W.; Corbett, M.A.; Gifford, W.D.; Gecz, J.; et al. Identification of a microRNA that activates gene expression by repressing nonsense-mediated RNA decay. Mol. Cell 2011, 42, 500-510.

35. Yepiskoposyan, H.; Aeschimann, F.; Nilsson, D.; Okoniewski, M.; Muhlemann, O. Autoregulation of the nonsense-mediated mRNA decay pathway in human cells. RNA 2011, 17, 2108-2118.

36. Huang, L.; Lou, C.H.; Chan, W.; Shum, E.Y.; Shao, A.; Stone, E.; Karam, R.; Song, H.W.; Wilkinson, M.F. RNA homeostasis governed by cell type-specific and branched feedback loops acting on NMD. Mol. Cell 2011, 43, 950-961.

37. Buchwald, G.; Ebert, J.; Basquin, C.; Sauliere, J.; Jayachandran, U.; Bono, F.; le Hir, H.; Conti, E. Insights into the recruitment of the NMD machinery from the crystal structure of a core EJC-UPF3B complex. Proc. Natl. Acad. Sci. USA 2010, 107, 10050-10055.

38. Gehring, N.H.; Neu-Yilik, G.; Schell, T.; Hentze, M.W.; Kulozik, A.E. Y14 and HUPF3B form an NMD-activating complex. Mol. Cell 2003, 11, 939-949.

39. Zund, D.; Gruber, A.R.; Zavolan, M.; Muhlemann, O. Translation-dependent displacement of UPF1 from coding sequences causes its enrichment in 3'-UTRs. Nat. Struct. Mol. Biol. 2013, 20, 936-943.

40. Hurt, J.A.; Robertson, A.D.; Burge, C.B. Global analyses of UPF1 binding and function reveal expanded scope of nonsense-mediated mRNA decay. Genome Res. 2013, 23, 1636-1650. 
41. Gehring, N.H.; Kunz, J.B.; Neu-Yilik, G.; Breit, S.; Viegas, M.H.; Hentze, M.W.; Kulozik, A.E. Exon-junction complex components specify distinct routes of nonsense-mediated mRNA decay with differential cofactor requirements. Mol. Cell 2005, 20, 65-75.

42. Kebaara, B.W.; Atkin, A.L. Long 3'-UTRs target wild-type mRNAs for nonsense-mediated mRNA decay in saccharomyces cerevisiae. Nucleic Acids Res. 2009, 37, 2771-2778.

43. Withers, J.B.; Beemon, K.L. The structure and function of the rous sarcoma virus RNA stability element. J. Cell. Biochem. 2011, 112, 3085-3092.

44. Kula, A.; Guerra, J.; Knezevich, A.; Kleva, D.; Myers, M.P.; Marcello, A. Characterization of the HIV-1 RNA associated proteome identifies matrin 3 as a nuclear cofactor of Rev function. Retrovirology 2011, doi:10.1186/1742-4690-8-60.

45. Milev, M.P.; Ravichandran, M.; Khan, M.F.; Schriemer, D.C.; Mouland, A.J. Characterization of staufen 1 ribonucleoproteins by mass spectrometry and biochemical analyses reveal the presence of diverse host proteins associated with human immunodeficiency virus type 1. Front. Microbiol. 2012, doi: $10.3389 /$ fmicb.2012.00367.

46. Hogg, J.R.; Goff, S.P. UPF1 senses 3'-UTR length to potentiate mRNA decay. Cell 2010, 143, 379-389.

47. Kunz, J.B.; Neu-Yilik, G.; Hentze, M.W.; Kulozik, A.E.; Gehring, N.H. Functions of HUPF3A and HUPF3B in nonsense-mediated mRNA decay and translation. RNA 2006, 12, 1015-1022.

48. Serin, G.; Gersappe, A.; Black, J.D.; Aronoff, R.; Maquat, L.E. Identification and characterization of human orthologues to saccharomyces cerevisiae UPF2 protein and UPF3 protein (caenorhabditis elegans SMG-4). Mol. Cell Biol. 2001, 21, 209-223.

49. Chan, W.K.; Bhalla, A.D.; le Hir, H.; Nguyen, L.S.; Huang, L.; Gecz, J.; Wilkinson, M.F. A UPF3-mediated regulatory switch that maintains RNA surveillance. Nat. Struct. Mol. Biol. 2009, 16, 747-753.

50. Okada-Katsuhata, Y.; Yamashita, A.; Kutsuzawa, K.; Izumi, N.; Hirahara, F.; Ohno, S. N- and C-terminal UPF1 phosphorylations create binding platforms for SMG-6 and SMG-5:SMG-7 during NMD. Nucleic Acids Res. 2012, 40, 1251-1266.

51. Truant, R.; Kang, Y.; Cullen, B.R. The human tap nuclear RNA export factor contains a novel transportin-dependent nuclear localization signal that lacks nuclear export signal function. J. Biol. Chem. 1999, 274, 32167-32171.

52. Wolff, H.; Hartl, A.; Eilken, H.M.; Hadian, K.; Ziegler, M.; Brack-Werner, R. Live-cell assay for simultaneous monitoring of expression and interaction of proteins. Biotechniques 2006, 41, 690-692.

53. Clerici, M.; Mourao, A.; Gutsche, I.; Gehring, N.H.; Hentze, M.W.; Kulozik, A.; Kadlec, J.; Sattler, M.; Cusack, S. Unusual bipartite mode of interaction between the nonsense-mediated decay factors, UPF1 and UPF2. EMBO J. 2009, 28, 2293-2306.

54. Dang, Y.; Low, W.K.; Xu, J.; Gehring, N.H.; Dietz, H.C.; Romo, D.; Liu, J.O. Inhibition of nonsense-mediated mRNA decay by the natural product pateamine a through eukaryotic initiation factor 4AIII. J. Biol. Chem. 2009, 284, 23613-23621.

55. Mendell, J.T.; Medghalchi, S.M.; Lake, R.G.; Noensie, E.N.; Dietz, H.C. Novel UPF2P orthologues suggest a functional link between translation initiation and nonsense surveillance complexes. Mol. Cell Biol. 2000, 20, 8944-8957. 
56. Monette, A.; Ajamian, L.; Lopez-Lastra, M.; Mouland, A.J. Human immunodeficiency virus type 1 (HIV-1) induces the cytoplasmic retention of heterogeneous nuclear ribonucleoprotein A1 by disrupting nuclear import: Implications for HIV-1 gene expression. J. Biol. Chem. 2009, 284, 31350-31362.

57. Chatel-Chaix, L.; Abrahamyan, L.; Frechina, C.; Mouland, A.J.; des Groseillers, L. The host protein staufen 1 participates in human immunodeficiency virus type 1 assembly in live cells by influencing pr55Gag multimerization. J. Virol. 2007, 81, 6216-6230.

58. Lehmann, M.; Milev, M.P.; Abrahamyan, L.; Yao, X.J.; Pante, N.; Mouland, A.J. Intracellular transport of human immunodeficiency virus type 1 genomic RNA and viral production are dependent on dynein motor function and late endosome positioning. J. Biol. Chem. 2009, 284, 14572-14585.

59. Beriault, V.; Clement, J.F.; Levesque, K.; Lebel, C.; Yong, X.; Chabot, B.; Cohen, E.A.; Cochrane, A.W.; Rigby, W.F.; Mouland, A.J. A late role for the association of hnRNP A2 with the HIV-1 hnRNP A2 response elements in genomic RNA, Gag, and Vpr localization. J. Biol. Chem. 2004, 279, 44141-44153.

60. Vyboh, K.; Ajamian, L.; Mouland, A.J. Detection of viral RNA by fluorescence in situ hybridization (fish). J. Vis. Exp. 2012, doi:10.3791/4002.

61. Abrahamyan, L.G.; Chatel-Chaix, L.; Ajamian, L.; Milev, M.P.; Monette, A.; Clement, J.F.; Song, R.; Lehmann, M.; Desgroseillers, L.; Laughrea, M.; et al. Novel staufen1 ribonucleoproteins prevent formation of stress granules but favour encapsidation of HIV-1 genomic RNA. J. Cell Sci. 2010, 123, 369-383.

62. Levesque, K.; Halvorsen, M.; Abrahamyan, L.; Chatel-Chaix, L.; Poupon, V.; Gordon, H.; DesGroseillers, L.; Gatignol, A.; Mouland, A.J. Trafficking of HIV-1 RNA is mediated by heterogeneous nuclear ribonucleoprotein A2 expression and impacts on viral assembly. Traffic 2006, 7, 1177-1193.

63. Jouvenet, N.; Bieniasz, P.D.; Simon, S.M. Imaging the biogenesis of individual HIV-1 virions in live cells. Nature 2008, 454, 236-240.

64. Chesebro, B.; Wehrly, K.; Nishio, J.; Perryman, S. Macrophage-tropic human immunodeficiency virus isolates from different patients exhibit unusual V3 envelope sequence homogeneity in comparison with T-cell-tropic isolates: Definition of critical amino acids involved in cell tropism. J. Virol. 1992, $66,6547-6554$.

65. Asai, K.; Platt, C.; Cochrane, A. Control of HIV-1 ENV RNA splicing and transport: Investigating the role of hnRNP A1 in exon splicing silencer (ESS3A) function. Virology 2003, 314, 229-242.

66. Mouland, A.J.; Mercier, J.; Luo, M.; Bernier, L.; DesGroseillers, L.; Cohen, E.A. The double-stranded RNA-binding protein staufen is incorporated in human immunodeficiency virus type 1: Evidence for a role in genomic RNA encapsidation. J. Virol. 2000, 74, 5441-5451.

67. Hogbom, M.; Collins, R.; van den Berg, S.; Jenvert, R.M.; Karlberg, T.; Kotenyova, T.; Flores, A.; Karlsson Hedestam, G.B.; Schiavone, L.H. Crystal structure of conserved domains 1 and 2 of the human Dead-box helicase DDX3X in complex with the mononucleotide AMP. J. Mol. Biol. 2007, $372,150-159$.

68. Di Mattia, M.A.; Watts, N.R.; Stahl, S.J.; Rader, C.; Wingfield, P.T.; Stuart, D.I.; Steven, A.C.; Grimes, J.M. Implications of the HIV-1 Rev dimer structure at 3.2 a resolution for multimeric binding to the Rev response element. Proc. Natl. Acad. Sci. USA 2010, 107, 5810-5814. 
69. Daugherty, M.D.; Booth, D.S.; Jayaraman, B.; Cheng, Y.; Frankel, A.D. HIV Rev response element (RRE) directs assembly of the Rev homooligomer into discrete asymmetric complexes. Proc. Natl. Acad. Sci. USA 2010, 107, 12481-12486.

70. Madhusudhan, M.S.; Webb, B.M.; Marti-Renom, M.A.; Eswar, N.; Sali, A. Alignment of multiple protein structures based on sequence and structure features. Protein Eng. Des. Sel. 2009, 22, 569-574.

71. Lyskov, S.; Chou, F.C.; Conchuir, S.O.; Der, B.S.; Drew, K.; Kuroda, D.; Xu, J.; Weitzner, B.D.; Renfrew, P.D.; Sripakdeevong, P.; et al. Serverification of molecular modeling applications: The rosetta online server that includes everyone (rosie). PLOS ONE 2013, 8, e63906.

72. Kortemme, T.; Joachimiak, L.A.; Bullock, A.N.; Schuler, A.D.; Stoddard, B.L.; Baker, D. Computational redesign of protein-protein interaction specificity. Nat. Struct. Mol. Biol. 2004, 11, 371-379.

73. Ajamian, L.; Mouland, A.J. McGill University, Montréal, Canada. Unpublished data, 2015.

74. Gong, C.; Kim, Y.K.; Woeller, C.F.; Tang, Y.; Maquat, L.E. SMD and NMD are competitive pathways that contribute to myogenesis: Effects on PAX3 and myogenin mRNAs. Genes Dev. 2009, $23,54-66$.

75. Nazarenus, T.; Cedarberg, R.; Bell, R.; Cheatle, J.; Forch, A.; Haifley, A.; Hou, A.; Kebaara, B.W.; Shields, C.; Stoysich, K.; et al. UPF1p, a highly conserved protein required for nonsense-mediated mRNA decay, interacts with the nuclear pore proteins NUP100P and NUP116P. Gene 2005, 345, 199-212.

76. Santos, S.; Obukhov, Y.; Nekhai, S.; Bukrinsky, M.; Iordanskiy, S. Virus-producing cells determine the host protein profiles of HIV-1 virion cores. Retrovirology 2012, doi:10.1186/1742-4690-9-65.

77. Applequist, S.E.; Selg, M.; Raman, C.; Jack, H.M. Cloning and characterization of HUPF1, a human homolog of the saccharomyces cerevisiae nonsense mRNA-reducing UPF1 protein. Nucleic Acids Res. 1997, 25, 814-821.

78. Dayton, A.I. Within you, without you: HIV-1 Rev and RNA export. Retrovirology 2004, doi:10.1186/ 1742-4690-1-35.

79. Suhasini, M.; Reddy, T.R. Cellular proteins and HIV-1 Rev function. Curr. HIV Res. 2009, 7, 91-100.

80. Milev, M.P.; Brown, C.M.; Mouland, A.J. Live cell visualization of the interactions between HIV-1 Gag and the cellular RNA-binding protein Staufen1. Retrovirology 2010, doi:10.1186/1742-4690-7-41.

81. Fornerod, M.; Ohno, M.; Yoshida, M.; Mattaj, I.W. CRM1 is an export receptor for leucine-rich nuclear export signals. Cell 1997, 90, 1051-1060.

82. Pierce, B.G.; Wiehe, K.; Hwang, H.; Kim, B.H.; Vreven, T.; Weng, Z. Zdock server: Interactive docking prediction of protein-protein complexes and symmetric multimers. Bioinformatics 2014, 30, 1771-1773.

83. Kadlec, J.; Guilligay, D.; Ravelli, R.B.; Cusack, S. Crystal structure of the UPF2-interacting domain of nonsense-mediated mRNA decay factor UPF1. RNA 2006, 12, 1817-1824.

84. De Turris, V.; Nicholson, P.; Orozco, R.Z.; Singer, R.H.; Muhlemann, O. Cotranscriptional effect of a premature termination codon revealed by live-cell imaging. RNA 2011, 17, 2094-2107.

85. Jager, S.; Cimermancic, P.; Gulbahce, N.; Johnson, J.R.; McGovern, K.E.; Clarke, S.C.; Shales, M.; Mercenne, G.; Pache, L.; Li, K.; et al. Global landscape of HIV-human protein complexes. Nature 2012, 481, 365-370. 
86. Serquina, A.K.; Das, S.R.; Popova, E.; Ojelabi, O.A.; Roy, C.K.; Gottlinger, H.G. UPF1 is crucial for the infectivity of human immunodeficiency virus type 1 progeny virions. J. Virol. 2013, 87, 8853-8861.

87. Booth, D.S.; Cheng, Y.; Frankel, A.D. The export receptor CRM1 forms a dimer to promote nuclear export of HIV RNA. eLife 2014, doi:10.7554/eLife.04121.

88. Sorokin, A.V.; Kim, E.R.; Ovchinnikov, L.P. Nucleocytoplasmic transport of proteins. Biochemistry 2007, 72, 1439-1457.

89. Zolotukhin, A.S.; Felber, B.K. Nucleoporins NUP98 and NUP214 participate in nuclear export of human immunodeficiency virus type 1 Rev. J. Virol. 1999, 73, 120-127.

90. Ariumi, Y.; Trono, D. Ataxia-telangiectasia-mutated (ATM) protein can enhance human immunodeficiency virus type 1 replication by stimulating Rev function. J. Virol. 2006, 80, 2445-2452.

91. Brumbaugh, K.M.; Otterness, D.M.; Geisen, C.; Oliveira, V.; Brognard, J.; Li, X.; Lejeune, F.; Tibbetts, R.S.; Maquat, L.E.; Abraham, R.T. The mRNA surveillance protein hSMG-1 functions in genotoxic stress response pathways in mammalian cells. Mol. Cell 2004, 14, 585-598.

92. Shiloh, Y. ATM and related protein kinases: Safeguarding genome integrity. Nat. Rev. Cancer 2003, 3, 155-168.

93. Kim, S.H.; Koroleva, O.A.; Lewandowska, D.; Pendle, A.F.; Clark, G.P.; Simpson, C.G.; Shaw, P.J.; Brown, J.W. Aberrant mRNA transcripts and the nonsense-mediated decay proteins UPF2 and UPF3 are enriched in the arabidopsis nucleolus. Plant Cell 2009, 21, 2045-2057.

94. Lejeune, F.; Ishigaki, Y.; Li, X.; Maquat, L.E. The exon junction complex is detected on CBP80-bound but not eIF4E-bound mRNA in mammalian cells: Dynamics of MRNP remodeling. EMBO J. 2002, $21,3536-3545$.

95. Maquat, L.E.; Gong, C. Gene expression networks: Competing mRNA decay pathways in mammalian cells. Biochem. Soc. Trans. 2009, 37, 1287-1292.

96. Shigeoka, T.; Kato, S.; Kawaichi, M.; Ishida, Y. Evidence that the UPF1-related molecular motor scans the 3'-UTR to ensure mRNA integrity. Nucleic Acids Res. 2012, 40, 6887-6897.

97. Chakrabarti, S.; Jayachandran, U.; Bonneau, F.; Fiorini, F.; Basquin, C.; Domcke, S.; le Hir, H.; Conti, E. Molecular mechanisms for the RNA-dependent atpase activity of UPF1 and its regulation by UPF2. Mol. Cell 2011, 41, 693-703.

(C) 2015 by the authors; licensee MDPI, Basel, Switzerland. This article is an open access article distributed under the terms and conditions of the Creative Commons Attribution license (http://creativecommons.org/licenses/by/4.0/). 